\title{
Influence of Variable Permeability and Radiation Absorption on Heat and Mass Transfer in MHD Micropolar Flow over a Vertical Moving Porous Plate
}

\author{
D. Harish Babu' ${ }^{1}$ and P. V. Satya Narayana ${ }^{2}$ \\ ${ }^{1}$ Department of Humanities and Basic Science, SVPCET, Puttur 517 583, India \\ ${ }^{2}$ Department of Mathematics, VIT University, Vellore 632 014, India \\ Correspondence should be addressed to P. V. Satya Narayana; pvsatya8@yahoo.co.in
}

Received 28 September 2012; Accepted 22 November 2012

Academic Editors: C. D. Daub, H. Hirao, D. E. Khoshtariya, and Z. Xu

Copyright (C) 2013 D. Harish Babu and P. V. Satya Narayana. This is an open access article distributed under the Creative Commons Attribution License, which permits unrestricted use, distribution, and reproduction in any medium, provided the original work is properly cited.

\begin{abstract}
An analysis of unsteady free convection with heat and mass transfer flow for a micropolar fluid through a porous medium with a variable permeability bounded by a semi-infinite vertical plate in the presence of heat generation, thermal radiation, first-order chemical reaction, and the radiation absorption is reported. The plate is assumed to move with a constant velocity in the direction of fluid flow. A uniform magnetic field acts perpendicular to the porous surface which absorbs micropolar fluid with a suction velocity varying with time. The dimensionless governing equations for this investigation are solved analytically using two-term harmonic and nonharmonic functions. To observe physical insight and interesting aspects of the problem, the velocity, angular velocity, temperature, and concentration field are numerically studied and displayed graphically for pertinent parameters.
\end{abstract}

\section{Introduction}

The study of heat and mass transfer for an electrically conducting micropolar fluid past a porous plate under the influence of a magnetic field has attracted the interest of many investigators in view of its applications in many engineering problems such as magnetohydrodynamic (MHD) generators, plasma studies, nuclear reactors, oil exploration, geothermal energy extractions, and the boundary layer control in the field of aerodynamics. The theory of microfluids that was developed by Eringen [1], which includes the effect of local rotary inertia, the couple stresses, and inertial spin, satisfactorily provides a model for the non-Newtonian behavior observed in polymers, paints, lubricants, suspended fluids and blood, and so forth. He [2] extended the theory of thermomicropolar fluids and derived the constitutive laws for fluids with microstructure. An excellent review of micropolar fluids and their applications was given by Ariman et al. [3]. Gorla [4] discussed the steady-state heat transfer in a micropolar fluid flow over a semi-infinite plate, and the analysis is based on similarity variables. Kim [5] studied unsteady free convection flow of micropolar fluids through a porous medium bounded by an infinite vertical plate. Charya [6] analyzed the flow of micropolar fluid through a constricted channel. Rees and Pop [7] discussed the free convection boundary layer flow of a micropolar fluid from a vertical flat plate, While Sharma and Gupta [8] studied the effects of medium permeability on thermal convection in micropolar fluids. Kumar et al. [9] have studied the problem of fully developed free convective flow of micropolar and viscous fluids in a vertical channel. Muthu et al. [10] studied peristaltic motion of micropolar fluid in circular cylindrical tubes. Srinivasacharya et al. [11] analyzed the unsteady stokes flow of micropolar fluid between two parallel porous plates. Recently, Muthuraj and Srinivas [12] investigated fully developed MHD flow of micropolar and viscous fluids in a vertical porous space using HAM.

The combined heat and mass transfer problems with chemical reactions are of importance in many processes and, therefore, have received a considerable amount of attention in recent years. In processes, such as drying, evaporation 
at the surface of a water body, energy transfer in a wet cooling tower, and the flow in a desert cooler, the heat and mass transfer occurs simultaneously. Das et al. [13] considered the effects of a first-order chemical reaction on the flow past an impulsively started infinite vertical plate with constant heat flux and mass transfer. The effects of the chemical reaction and mass transfer on MHD unsteady free convection flow past an infinite/semi-infinite vertical plate were analyzed by [14-17] with constant suction and heat sink. Reddy et al. [18] studied peristaltic transport of a conducting fluid in an inclined asymmetric channel. Recently, Pal and Talukdar [19] studied the unsteady magnetohydrodynamic convective heat and mass transfer in a boundary layer slip flow past a vertical permeable plate with thermal radiation and chemical reaction.

Convection problems associated with heat sources within fluid-saturated porous media are of great practical significance, for that there are a number of practical applications in geophysics and energy-related problems, such as recovery of petroleum resources, geophysical flows, cooling of underground electric cables, storage of nuclear waste materials ground water pollution, fiber and granular insulations, solidification of costing, chemical catalytic reactors, and environmental impact of buried heat generating waste. Abo-Eldahab and El Aziz [20] investigated the problem of steady, laminar, free convection boundary layer flow of a micropolar fluid from a vertical stretching surface embedded in a nondaring porous medium in the presence of a uniform magnetic field, heat generation/absorption, and free stream velocity. Gorla et al. [21] have presented an analysis for the unsteady natural convection from a heated vertical surface placed in a micropolar fluid in the presence of internal heat generation or absorption. Raptis and Perdikis [22] analyzed the effect of a chemical reaction of an electrically conducting viscous fluid on the flow over a nonlinearly (quadratic) semi-infinite stretching sheet in the presence of a constant magnetic field which is normal to the sheet. Kesavaiah et al. [23] investigated the effects of the chemical reaction and radiation absorption on an unsteady MHD convective heat and mass transfer flow past a semi-infinite vertical permeable moving plate. Recently, Vajravelu et al. [24] studied the influence of heat transfer on peristaltic transport of a Jeffrey fluid in a vertical porous stratum.

The role of thermal radiation in the flow heat transfer process is of major importance in the design of many advanced energy conversion systems operating at higher temperatures. Thermal radiation within these systems is usually the result of emission by hot walls and the working fluid. Bakier and Gorla [25] studied thermal radiation effect on mixed convection from horizontal surfaces in porous media. Bakier [26] reported the effect of radiation on the mixed convection flow on an isothermal vertical surface in a saturated porous medium and has obtained self-similar solution. Hossain and Takhar [27] analyzed the effect of radiation on mixed convection along a vertical plate with uniform surface temperature.

In many investigations, while investigating either velocity and temperature distribution or stability characteristics of the flow usually the permeability and porosity are taken as constants. All the above-mentioned studies treat permeability and thermal conductivity of the medium as uniform. It has been observed that when porosity and permeability are taken as constants, the velocity profiles are flat with a thin boundary near the walls. However, the experimental investigations of Schwartz and Smith [28] and Schertz and Bischoff [29] show that the velocity profiles exhibit channeling of velocity near the walls. This hydrodynamic channeling is attributed to the variation of porosity of the medium confined between two boundaries. Further, the measurements of porosity distribution by Benenati and Brosilow [30] in randomly packed beds established a graphical relationship between spatial dependence of porosity and radial position of the container from which we find that the porosity has a maximum value at the wall and minimum value at the center of the bed. Thus, it can be concluded that the porosity and in turn permeability are not constant in a given medium. However, there is no exact mathematical relationship between the porosity variation and the distance from the wall. Chandrasekhara and Namboodiri [31] incorporate the variable permeability concept. They indicated considerable effect on the flow velocity and temperature distributions. Murthy and Feyen [32] studied the influence of variable permeability on the dispersion of a chemically reacting solute in porous media. From the technological point of view, flow arising from temperature and material difference is applied in chemical engineering, geothermal reservoirs, aeronautics, and astrophysics. In some applications, magnetic forces are present and at other times the flow is further complicated by the presence of radiation absorption, a good example of this is in the planetary atmosphere where there is radiation absorption from nearby stars. The influence of a magnetic field on the flow of an electrically conducting viscous fluid with mass transfer and radiation absorption is also useful in planetary atmosphere research [33]. The effect of chemical reaction and radiation absorption on the unsteady MHD free convection flow past a semi-infinite vertical permeable moving plate with heat source and suction was analyzed by $[34,35]$.

To the best of our knowledge, the problem of magnetohydrodynamics unsteady free convection flow of a micropolar fluid through a porous medium of variable permeability with radiation absorption over a vertical moving porous flat plate has remained unexplored. So the objective of present paper is to study the effects of a first-order homogeneous chemical reaction, variable permeability, and thermal radiation on the heat and mass transfer in MHD micropolar fluid flow over a vertical moving porous flat plate through a porous medium.

\section{Formulation}

We consider the two-dimensional unsteady flow of a laminar, incompressible micropolar fluid past a semi-infinite vertical porous moving plate embedded in a porous medium with variable permeability. The $x^{*}$-axis is measured along the porous plate in the upward direction, and a magnetic field of uniform strength $B_{0}$ is applied in the $y^{*}$-direction which is normal to the flow direction. Since the motion is twodimensional and length of the plate is very large, so all the physical variables are independent of $x^{*}$ and they are functions of normal distance $y^{*}$ and $t^{*}$ only. It is assumed that 
transversely applied magnetic field and magnetic Reynolds number are very small so that the induced magnetic field and the Hall effect are negligible. The motion of the plate as well as permeability of the porous medium and suction velocity increases exponentially with time. The wall is maintained at constant temperature $T_{w}^{*}$ and concentration $C_{w}^{*}$, higher than the ambient temperature $T_{\infty}^{*}$ and concentration $C_{\infty}^{*}$, respectively. The permeability of the porous medium is assumed to be of the form $K^{*}=K_{0}\left(1+B \varepsilon e^{\delta^{*} t^{*}}\right)$ and suction velocity is assumed to be $v^{*}=-V_{0}\left(1+\varepsilon A e^{\delta^{*} t^{*}}\right)$, where $A$, $B$ are real positive constants and $\varepsilon$ and $\varepsilon A$ are small values less than unity, and $V_{0}$ is the scale of suction velocity which is nonzero positive constant. The negative sign indicates that the suction is towards the plate. The fluid properties are assumed to be constants except that the influence of density variation with temperature and concentration has been considered in the body-force term. The concentration of diffusing species is very small in comparison to other chemical species; the concentration of species far from the wall $C_{\infty}^{*}$ is infinitesimally small and hence the Soret and Dufour effects are neglected. The chemical reactions are taking place in the flow and all thermophysical properties are assumed to be constant.

Under these conditions, the governing equations can be written in a Cartesian frame of reference as follows:

$$
\begin{gathered}
\frac{\partial v^{*}}{\partial y^{*}}=0 \\
\frac{\partial u^{*}}{\partial t^{*}}+v^{*} \frac{\partial u^{*}}{\partial y^{*}}=-\frac{1}{\rho} \frac{\partial P^{*}}{\partial x^{*}} \\
+\left(v+v_{r}\right) \frac{\partial^{2} u^{*}}{\partial y^{* 2}}+g \beta_{f}\left(T^{*}-T_{\infty}^{*}\right) \\
+g \beta_{c}\left(C^{*}-C_{\infty}^{*}\right) \\
-v \frac{u^{*}}{K_{0}\left(1+B \varepsilon e^{\delta^{*} t^{*}}\right)}-\frac{\sigma}{\rho} B_{0}^{2} u^{*}+2 v_{r} \frac{\partial \omega^{*}}{\partial y^{*}} \\
\rho j^{*}\left(\frac{\partial \omega^{*}}{\partial t^{*}}+v^{*} \frac{\partial \omega^{*}}{\partial y^{*}}\right)=\gamma \frac{\partial^{2} \omega^{*}}{\partial y^{* 2}} \\
\frac{\partial T^{*}}{\partial t^{*}}+v^{*} \frac{\partial T^{*}}{\partial y^{*}}=\frac{K}{\rho c_{p}} \frac{\partial^{2} T^{*}}{\partial y^{* 2}}+\frac{Q_{0}}{\rho c_{p}}\left(T^{*}-T_{\infty}^{*}\right) \\
+Q_{l}^{*}\left(C^{*}-C_{\infty}^{*}\right)-\frac{1}{\rho c_{p}^{*}} \frac{\partial q_{r}^{*}}{\partial y^{*}} \\
+v^{*} \frac{\partial c^{*}}{\partial y^{*}}=D^{*} \frac{\partial^{2} c^{*}}{\partial y^{* 2}}-R^{*}\left(C^{*}-C_{\infty}^{*}\right)
\end{gathered}
$$

The third and fourth terms on the RHS of the momentum equation (2) denote the thermal and concentration buoyancy effects, respectively. The second, third, and fourth terms on RHS of (3) denote the heat absorption, radiation absorption, and thermal radiation effects, respectively.
Under these assumptions, the appropriate boundary conditions for the velocity, angular velocity, temperature, and concentration fields are

$$
\begin{gathered}
u^{*}=u_{p}^{*}, \quad T^{*}=T_{w}^{*}+\varepsilon\left(T_{w}^{*}-T_{\infty}^{*}\right) e^{\delta^{*} t^{*}}, \\
\omega^{*}=-n \frac{\partial u^{*}}{\partial y^{*}}, \\
C^{*}=C_{w}^{*}+\varepsilon\left(C_{w}^{*}-C_{\infty}^{*}\right) e^{\delta^{*} t^{*}} \text { at } y \longrightarrow 0, \\
u^{*} \longrightarrow U_{\infty}^{*}=U_{0}\left(1+\varepsilon e^{\delta^{*} t^{*}}\right), \\
T^{*} \longrightarrow T_{\infty}^{*}, \quad C^{*} \longrightarrow C_{\infty}^{*}, \\
\omega^{*} \longrightarrow 0 \quad \text { at } y \longrightarrow \infty,
\end{gathered}
$$

where the variables and related quantities are defined in the nomenclature.

Outside the boundary layer, (2) gives

$$
-\frac{1}{\rho} \frac{\partial P^{*}}{\partial x^{*}}=\frac{d U_{\infty}^{*}}{d t^{*}}+\frac{v}{K^{*}} U_{\infty}^{*}+\frac{\sigma}{\rho} B_{0}^{2} U_{\infty}^{*} .
$$

The radiative heat flux term by using the Rosseland approximations is given by

$$
q_{r}^{*}=\frac{4 \sigma^{*}}{3 K_{1}^{*}} \frac{\partial T^{* 4}}{\partial y^{*}},
$$

where $T^{* 4}=4 T_{\infty}^{* 3}-3 T_{\infty}^{* 4}$.

In order to write the governing equations and the boundary conditions in dimensionless form, the following nondimensional quantities are introduced:

$$
\begin{gathered}
u^{*}=U_{0} u, \quad v^{*}=V_{0} v, \quad u_{p}^{*}=U_{0} U_{p}, \\
y^{*}=\frac{v y}{V_{0}}, \quad U_{\infty}^{*}=U_{0} U_{\infty}, \\
\omega^{*}=\frac{\omega U_{0} V_{0}}{V_{0}}, \quad j^{*}=\frac{j v^{2}}{V_{0}^{2}}, \quad t^{*}=\frac{t v}{V_{0}^{2}}, \\
c^{*}=c_{\infty}^{*}+c\left(c_{w}^{*}-c_{\infty}^{*}\right), \quad \delta^{*}=\frac{\delta v_{0}^{*}}{v}, \\
K^{*}=\frac{K v_{0}^{2}}{V_{0}^{2}}, \quad s_{c}=\frac{v}{D^{*}}, \quad H=\frac{v Q_{0}}{\rho v c_{p}}, \\
\zeta=\frac{R^{*} v}{V_{0}^{2}}, \quad Q_{l}^{*}=\frac{Q_{l}^{*} C\left(C_{w}^{*}-C_{\infty}^{*}\right)}{\left(T_{w}^{*}-T_{\infty}^{*}\right) V_{0}^{2}}, \\
\mathrm{Gr}=\left(\frac{v g B_{0}^{2}}{U_{0} V_{0}^{2}}\right)\left(T_{w}^{*}-T_{\infty}^{*}\right) \text { is the thermal Grashof number, } \\
M V_{0}^{2}
\end{gathered}
$$


$\mathrm{Gc}=\frac{v g B_{c}}{U_{0} V_{0}^{2}}\left(c_{w}^{*}-c_{\infty}^{*}\right)$ is the solutal Grashof number, $\operatorname{Pr}=\frac{v \rho c_{p}}{K}=\frac{v}{\alpha}$ is the Prandtl number, $R=\frac{4 T_{\infty}^{* 3} \sigma^{*}}{K K_{1}^{*}}$ is the radiation parameter, $\alpha=\frac{K}{\rho c_{p}}$ is the effective fluid thermal diffusivity.

Furthermore, the spin-gradient viscosity, which gives the same relationship between the coefficients of viscosity and microinertia, is defined as

$$
\gamma=\left(\mu+\frac{\Gamma}{2}\right) j^{*}=\mu j^{*}\left(1+\frac{\beta}{2}\right),
$$

where $\beta=\Gamma / \mu$.

In view of (7)-(10), the governing equations (2)-(5) reduce to the following dimensionless form:

$$
\begin{gathered}
\frac{\partial u}{\partial t}-\left(1+\varepsilon A e^{\delta t}\right) \frac{\partial u}{\partial y} \\
=\frac{d u_{\infty}}{d t}+(1+\beta) \frac{\partial^{2} u}{\partial y^{2}}+\mathrm{Gr} \theta+\mathrm{GcC} \\
+\frac{\left(U_{\infty}-u\right)}{K\left(1+B \varepsilon e^{\delta t}\right)}+M\left(U_{\infty}-u\right)+2 \beta \frac{\partial \omega}{\partial y}, \\
\frac{\partial \omega}{\partial t}-\left(1+\varepsilon A e^{\delta t}\right) \frac{\partial \omega}{\partial y}=\frac{1}{\eta} \frac{\partial^{2} \omega}{\partial y^{2}}, \\
\frac{\partial \theta}{\partial t}-\left(1+\varepsilon A e^{\delta t}\right) \frac{\partial \theta}{\partial y} \\
=\frac{1}{\operatorname{Pr}}\left(1+\frac{4 R}{3}\right) \frac{\partial^{2} \theta}{\partial y^{2}}+H \theta+Q_{l} c \\
\frac{\partial c}{\partial t}-\left(1+\varepsilon A e^{\delta t}\right) \frac{\partial c}{\partial y}=\frac{1}{\mathrm{Sc}} \frac{\partial^{2} c}{\partial y^{2}}-\zeta c
\end{gathered}
$$

where $\eta=2 / 2+\beta=\mu j^{*} / \gamma$.

The boundary conditions of (6) are given by the following dimensionless form:

$$
\begin{gathered}
u=U_{p}, \quad \theta=1+\varepsilon e^{\delta t}, \quad \omega=-n \frac{\partial u}{\partial y}, \\
c=1+\varepsilon e^{\delta t} \quad \text { at } y \longrightarrow 0, \\
u=U_{\infty} \longrightarrow\left(1+\varepsilon e^{\delta t}\right), \quad \theta \longrightarrow 0, \\
\omega \longrightarrow 0, \quad c \longrightarrow 0 \quad \text { at } y \longrightarrow \infty
\end{gathered}
$$

\section{Solution of the Problem}

Equations (11)-(14) are coupled, nonlinear partial differential equations and these cannot be solved in closed form. However, these equations can be reduced to ordinary differential equations, which can be solved analytically. For these, we may represent the linear and angular velocities, temperature, and concentration as follows:

$$
\begin{aligned}
& u=u_{0}(y)+\varepsilon e^{\delta t} u_{1}(y)+o\left(\varepsilon^{2}\right), \\
& \omega=\omega_{0}(y)+\varepsilon e^{\delta t} \omega_{1}(y)+o\left(\varepsilon^{2}\right), \\
& \theta=\theta_{0}(y)+\varepsilon e^{\delta t} \theta_{1}(y)+o\left(\varepsilon^{2}\right), \\
& c=c_{0}(y)+\varepsilon e^{\delta t} c_{1}(y)+o\left(\varepsilon^{2}\right) .
\end{aligned}
$$

By substituting the above equations (16) into (11)-(14), equating the harmonic and nonharmonic terms, and neglecting the higher-order terms of $O\left(\varepsilon^{2}\right)$, we obtain the following pairs of equations for $\left(u_{0}, \omega_{0}, \theta_{0}, C_{0}\right)$ and $\left(u_{1}, \omega_{1}, \theta_{1}, C_{1}\right)$ :

$$
\begin{aligned}
& (1+\beta) u_{0}^{\prime \prime}+u_{0}^{\prime}-N u_{0} \\
& =-N-\operatorname{Gr} \theta_{0}-\mathrm{Gcc} c_{0}-2 \beta \omega_{0}^{\prime}, \\
& (1+\beta) u_{1}^{\prime \prime}+u_{1}^{\prime}-(N+\delta) u_{1} \\
& =-L u_{0}+L-\operatorname{Gr} \theta_{1}-\mathrm{Gcc} c_{1}-2 \beta \omega_{1}^{\prime}-A u_{0}^{\prime}, \\
& \omega_{0}^{\prime \prime}+\eta \omega_{0}=0 \\
& \omega_{1}^{\prime \prime}+\eta \omega_{1}^{\prime}-\delta \eta \omega_{1}=-A \eta \omega_{0}^{\prime}, \\
& (3+4 R) \theta_{0}^{\prime \prime}+3 \operatorname{Pr}\left(\theta_{0}^{\prime}+H \theta_{0}\right)=-3 \operatorname{Pr} Q_{l} c_{0}, \\
& (3+4 R) \theta_{1}^{\prime \prime}+3 \operatorname{Pr} \theta_{1}^{\prime}-3 \operatorname{Pr}(\delta-H) \theta_{1} \\
& =-3 A \operatorname{Pr} \theta_{0}^{\prime}-3 \operatorname{Pr} Q_{l} c_{1}, \\
& c_{0}^{\prime \prime}+\operatorname{Scc} c_{0}^{\prime}-\zeta \operatorname{Scc} c_{0}=0, \\
& c_{1}^{\prime \prime}+\operatorname{Scc}_{1}^{\prime}-(\delta+\zeta) \operatorname{Scc}=-A S c c_{0}^{\prime},
\end{aligned}
$$

where $L=B / K, N=(M+1 / K)$ and the primes denote differentiation with respect to $y$.

The corresponding boundary conditions can be written as

$$
\begin{gathered}
u_{0}=U_{p}, \quad u_{1}=0, \quad \omega_{0}=-n u_{0}^{\prime}, \quad \omega_{1}=-n u_{1}^{\prime}, \\
\theta_{0}=1, \quad \theta_{1}=1, \quad c_{0}=1, \quad c_{1}=1 \quad \text { at } y \longrightarrow 0, \\
u_{0}=1, \quad u_{1}=1, \quad \omega_{0}=0, \quad \omega_{1}=0, \\
\theta_{0}=0, \quad \theta_{1}=0, \quad c_{0}=0, \quad c_{1}=0 \quad \text { at } y \longrightarrow \infty .
\end{gathered}
$$


TABLE 1: Effect of $\operatorname{Pr}$ on $C_{f}$, Nu and Sh for $R=0.1, n=0.5, K=2, H=0.1, \zeta=0.2, \beta=0.5, \mathrm{Sc}=0.2, \operatorname{Pr}=1, \mathrm{Gr}=2, U_{p}=0.5, Q_{l}=0.01$, $B=0.01$, and $\mathrm{Gc}=2$.

\begin{tabular}{|c|c|c|c|c|c|c|c|c|c|}
\hline \multirow[t]{2}{*}{$\operatorname{Pr}$} & \multicolumn{3}{|c|}{ Kim [5] } & \multicolumn{3}{|c|}{$\begin{array}{c}\text { Mohamed and } \\
\text { Abo-Dahab [36] } \\
Q_{l}=0 \text { and } B=0\end{array}$} & \multicolumn{3}{|c|}{ Present Results } \\
\hline & $C_{f}$ & $\mathrm{Nu}$ & $\mathrm{Sh}$ & $C_{f}$ & $\mathrm{Nu}$ & $\mathrm{Sh}$ & $C_{f}$ & $\mathrm{Nu}$ & $\mathrm{Sh}$ \\
\hline 2 & 5.6985 & 20.6907 & 0.3276 & 5.6964 & 20.6897 & 0.3276 & 5.6933 & 20.6899 & 0.3276 \\
\hline 3 & 5.6316 & 31.9168 & 0.3276 & 5.6304 & 33.9140 & 0.3276 & 5.6300 & 31.9155 & 0.3276 \\
\hline 4 & 5.5972 & 43.1064 & 0.3276 & 5.5967 & 43.1001 & 0.3276 & 5.5943 & 43.1034 & 0.3276 \\
\hline 5 & 5.5763 & 54.3059 & 0.3276 & 5.5752 & 54.3000 & 0.3276 & 5.5751 & 54.3038 & 0.3276 \\
\hline
\end{tabular}

TABLE 2: Effect of $M$ on $C_{f}$ and Sh for $R=0.1, n=0.5, K=2, H=0.1, \zeta=0.2, \beta=0.5, \mathrm{Sc}=0.2, \operatorname{Pr}=1, \mathrm{Gr}=2, U_{p}=0.5, Q_{l}=0.01$, $B=0.01$, and $\mathrm{Gc}=2$.

\begin{tabular}{|c|c|c|c|c|c|c|}
\hline \multirow{3}{*}{$M$} & \multicolumn{2}{|c|}{ Kim [5] } & \multicolumn{4}{|c|}{ Mohamed and Abo-Dahab [36] } \\
\hline & \multicolumn{2}{|c|}{$H=0, Q_{l}=0, R=0$, and $B=0$} & \multicolumn{2}{|c|}{$Q_{l}=0$ and $B=0$} & \multicolumn{2}{|c|}{ Present results } \\
\hline & $C_{f}$ & Sh & $C_{f}$ & $\mathrm{Sh}$ & $C_{f}$ & $\mathrm{Sh}$ \\
\hline 1 & 6.4837 & 0.3276 & 6.4892 & 0.3276 & 6.4893 & 0.3276 \\
\hline 3 & 5.6678 & 0.3276 & 5.6685 & 0.3276 & 5.6686 & 0.3276 \\
\hline 4 & 5.5941 & 0.3276 & 5.5986 & 0.3276 & 5.5987 & 0.3276 \\
\hline 5 & 5.5894 & 0.3276 & 5.5800 & 0.3276 & 5.5804 & 0.3276 \\
\hline
\end{tabular}

Solving (17) under the boundary conditions (18), we obtain the linear and angular velocities, temperature, and concentration profiles as follows:

$$
\begin{aligned}
u(y, t)= & \left(1+a_{1} e^{-R_{1} y}+a_{2} e^{-h_{3} y}+a_{6} e^{-h_{1} y}+a_{5} e^{-\eta y}\right) \\
& +\varepsilon e^{\delta t}\left(1+b_{1} e^{-R_{3} y}+b_{2} e^{-h_{4} y}+d_{1} e^{-h_{3} y}+d_{2} e^{-h_{2} y}\right. \\
& \left.+d_{3} e^{-h_{1} y}+b_{8} e^{-h_{6} y}+b_{10} e^{-R_{1} y}+d_{4} e^{-\eta y}\right), \omega(y, t) \\
= & \left(c_{11} e^{-\eta y}\right)+\varepsilon e^{\delta t}\left(c_{13} e^{-h_{6} y}-\frac{A \eta c_{11}}{\delta} e^{-\eta y}\right), \theta(y, t) \\
= & \left(\left(1-z_{3}\right) e^{-h_{3} y}+z_{3} e^{-h_{1} y}\right) \\
& +\varepsilon e^{\delta t}\left(c_{9} e^{-h_{4} y}+z_{4} e^{-h_{3} y}+z_{6} e^{-h_{2} y}+z_{8} e^{-h_{1} y}\right), c(y, t) \\
= & e^{-h_{1} y}+\varepsilon e^{\delta t}\left(\left(1-z_{3}\right) e^{-h_{2} y}+z_{2} e^{-h_{1} y}\right),
\end{aligned}
$$

where the expressions for the constants are given in the appendix.

The skin friction, Nusselt number, and Sherwood number are important physical parameters for this type of boundary layer.

3.1. Skin-Friction. Knowing the velocity field in the boundary layer, we can now calculate the skin-friction at the plate which is given by

$$
\tau_{w}^{*}=(\mu+\Gamma) \frac{\partial u^{*}}{\partial y^{*}}+\left.\Gamma \omega^{*}\right|_{y^{*}=0},
$$

and in the dimensionless form, we obtain

$$
c_{f}=\frac{2 \tau_{w}^{*}}{\rho V_{0} U_{0}}=2[1+(1-n) \beta] u^{\prime}(0) .
$$

The couple stress coefficient $\left(C_{m}\right)$ at the plate is written as

$$
M_{w}=\left.\gamma \frac{\partial \omega^{*}}{\partial y^{*}}\right|_{y^{*}=0}
$$

and in the dimensionless from, we obtain

$$
c_{m}=\frac{M_{w}}{\mu j U_{0}}=\left(1+\frac{\beta}{2}\right) \omega^{\prime}(0) .
$$

3.2. Nusselt Number. Knowing the temperature field, it is interesting to study the effect of the free convection and thermal radiation on the rate of heat transfer $q_{w}^{*}$, and this is given by

$$
q_{w}^{*}=-k\left(\frac{\partial T^{*}}{\partial y^{*}}\right)-\left.\frac{4 \sigma^{*}}{3 K_{1}^{*}}\left(\frac{\partial T^{* 4}}{\partial y^{*}}\right)\right|_{y^{*}=0} .
$$

In view of (8), the above equation becomes

$$
q_{w}^{*}=\left.\left(-k+\frac{16 \sigma^{*} T^{* 3}}{3 K_{1}^{*}}\right) \frac{\partial T^{*}}{\partial y^{*}}\right|_{y^{*}=0} .
$$

This is written in dimensionless from as

$$
q_{w}^{*}=\left.\left(\frac{-k\left(T_{w}^{*}-T_{\infty}^{*}\right) v_{0}}{v}\right)\left(1+\frac{4 R}{3}\right) \frac{\partial \theta}{\partial y}\right|_{y=0} .
$$


The dimensionless local surface heat flux (i.e., Nusselt number) is obtained as

$$
\mathrm{Nu}_{x}=\frac{x q_{w}^{*}}{k\left(T_{w}^{*}-T_{\infty}^{*}\right)},
$$

where

$$
\begin{gathered}
\mathrm{Nu}_{x} \mathrm{Re}_{x}^{-1}=-\left.\left(1+\frac{4 R}{3}\right) \frac{\partial \theta}{\partial y}\right|_{y=0}, \\
\operatorname{Re}_{x}=\frac{v_{0} x}{v}
\end{gathered}
$$

3.3. Sherwood Number. The definition of the local mass flux and the local Sherwood number are, respectively, given by

$$
\begin{gathered}
j_{w}=-\left.D^{*}\left(\frac{\partial c^{*}}{\partial y^{*}}\right)\right|_{y^{*}=0}, \\
\mathrm{Sh}_{x}=\frac{x j_{w}}{D^{*}\left(c_{w}^{*}-c_{w}^{*}\right)}
\end{gathered}
$$

and with the help of these equations, one can write

$$
\mathrm{Sh}_{x} \operatorname{Re}_{x}^{-1}=-\left.\left(\frac{\partial c}{\partial y}\right)\right|_{y=0}
$$

\section{Results and Discussions}

The formulation of the problem that accounts for the effects of chemical reaction and thermal radiation on MHD convective flow and mass transfer of an incompressible, micropolar fluid along a semi-infinite vertical porous moving plate in a porous medium with variable permeability in the presence of heat generation has been performed in the preceding sections. The governing equations of the flow field were solved analytically, using a perturbation method, and the expressions for the velocity, microrotation, temperature, concentration, skin-friction, Nusselt number, and Sherwood number were obtained. In order to get a physical insight of the problem, the above physical quantities are computed numerically and presented graphically for different values of the governing parameters: thermal Grashof number Gr, solutal Grashof number $\mathrm{Gm}$, magnetic field parameter $M$, Schmidt number Sc, heat absorption parameter $H$, chemical reaction parameter $\zeta$, and thermal radiation parameter $R$, while the values of some of the physical parameters are taken as constants such as $t=1, \delta=0.01, \varepsilon=0.01, B=0.1$ and $A=0.1$, in all the tables and figures. In the present study, the boundary conditions for $y \rightarrow \infty$ are replaced by identical ones at $y_{\max }$ which is a sufficiently large value of $y$ where the velocity profile $u$ approaches the relevant free stream velocity. We choose $y_{\max }=8$ and a step size $\Delta y=0.001$.

In order to verify the accuracy of the present work, particular results are compared with those available in the literature through Table 1 . The numerical values of skinfriction, Nusselt number, and Sherwood number obtained in the present case are compared with those of Kim [5] and
Mohamed and Abo-Dahab [36] for different values of Prandtl number $(\mathrm{Pr})$ and fixed values of remaining parameters. It is clearly seen that there is an excellent agreement between the respective research of our study and those of Kim [5] and Mohamed and Abo-Dahab [36]. Further, it is found from Table 2 that skin-friction decreases with the increase in magnetic parameter $M$. Also no effect of $M$ is seen on Sherwood number Sh as noted in [36].

For different values of the magnetic field parameter $M$, the translational velocity and microrotation profiles are plotted in Figure 1. It is obvious that the effect of increasing values of the parameter $M$ results in a decreasing velocity distribution across the boundary layer. The effect of magnetic field is more prominent at the point of peak value; that is, the peak value drastically decreases with the increases in the value of magnetic field because the presence of magnetic field in an electrically conducting fluid introduces a force called the Lorentz force, which acts against the flow if the magnetic field is applied in the normal direction, as in the present problem. This type of resisting force slows down the fluid velocity as shown in this figure. The results also show that the magnitude of the microrotation on the porous plate increases as $M$ increases.

Figure 2 illustrates the variation of velocity and microrotation distribution across the boundary layer for several values of plate moving velocity $U_{p}$ in the direction of the fluid flow, respectively. Although we have different initial plate moving velocities, the velocity decreases to the constant values for given material parameters. The results also show that the magnitude of microrotation on porous plate increases as $U_{p}$ increases.

For various values of the permeability $K$, the profiles of translational velocity and the microrotation across the boundary layer are shown in Figure 3. It is clear that as $K$ increase, the translational velocity profiles across the boundary layer increases, but the magnitude of microrotation decreases with an increase of $K$-parameter.

Figure 4 presents the velocity and microrotation profiles for different values of the Prandtl number (Pr). The results show that the effect of increasing values of Pr results in a decrease of the translational velocity, while the reverse effect is observed for the microrotation profiles.

For various values of the Schmidt number (Sc), the profiles of translational velocity and the microrotation across the boundary layer are shown in Figure 5. It is clear that as $\mathrm{Sc}$ increase, the translational velocity profiles across the boundary layer decreases. The results also show that the magnitude of microrotation on porous plate increases as Sc increases.

For various values of $Q_{l}$, the profiles of translational velocity and the microrotation across the boundary layer are shown in Figure 6. It is clear from the graph that the translational velocity increases with an increase of $Q_{l}$ which is due to the fact that when heat is absorbed the buoyancy force which accelerated the flow rate. The results also show that the magnitude of microrotation on porous plate decreases as $Q_{l}$ increases.

For various values of radiation parameter $R$, the translational velocity and microrotation profiles are plotted in 


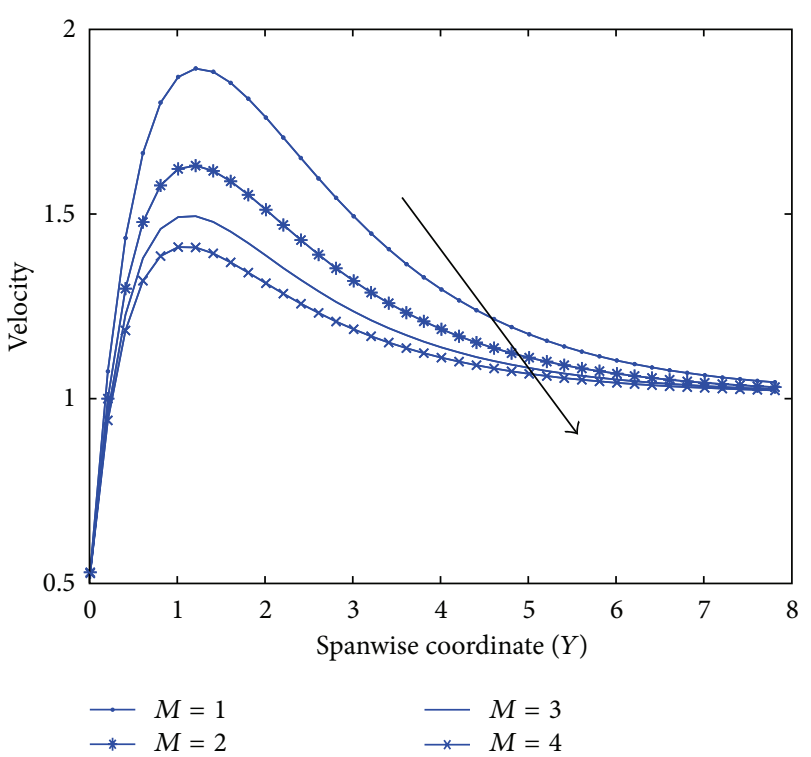

(a)

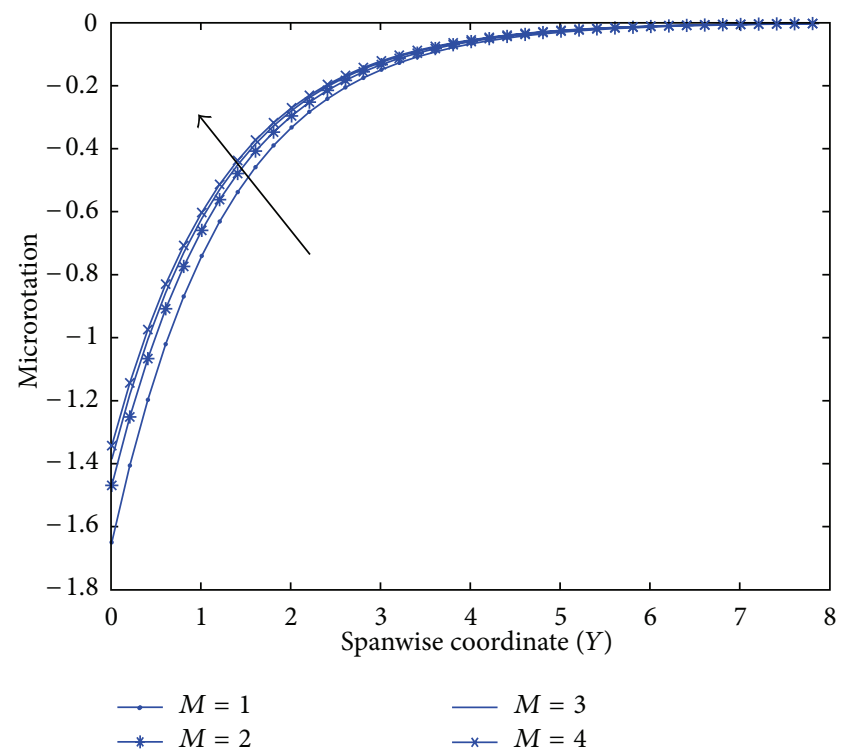

(b)

FIGURE 1: Velocity and microrotation distribution.

Figure 7. It is obvious that with increasing values of $R$ parameter, the velocity distribution across the boundary layer increases. The results also show that the magnitude of microrotation on the porous plate is decreased as $R$-parameter increases.

The effects of heat generation $H$ on the translational velocity and microrotation profiles across the boundary layer are displayed in Figure 8. It is shown that the translational velocity across the boundary layer increases with an increasing of $H$. The results also show that the magnitude of microrotation decreases with an increasing of $H$.

Figure 9 shows the translational velocity and the microrotation profiles across the boundary layer for various values of the chemical reaction parameter $\zeta$. The results display that

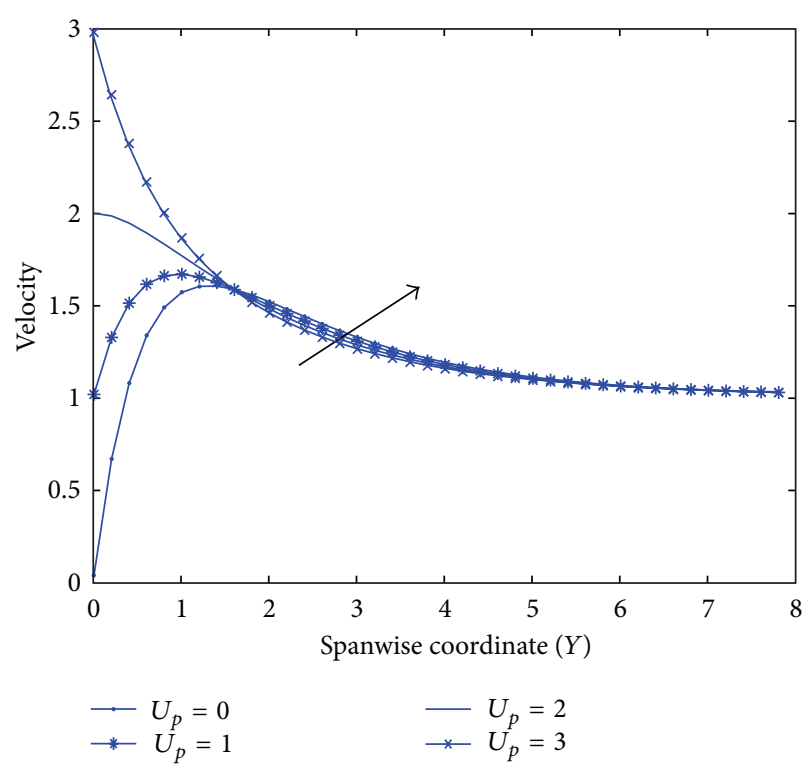

(a)

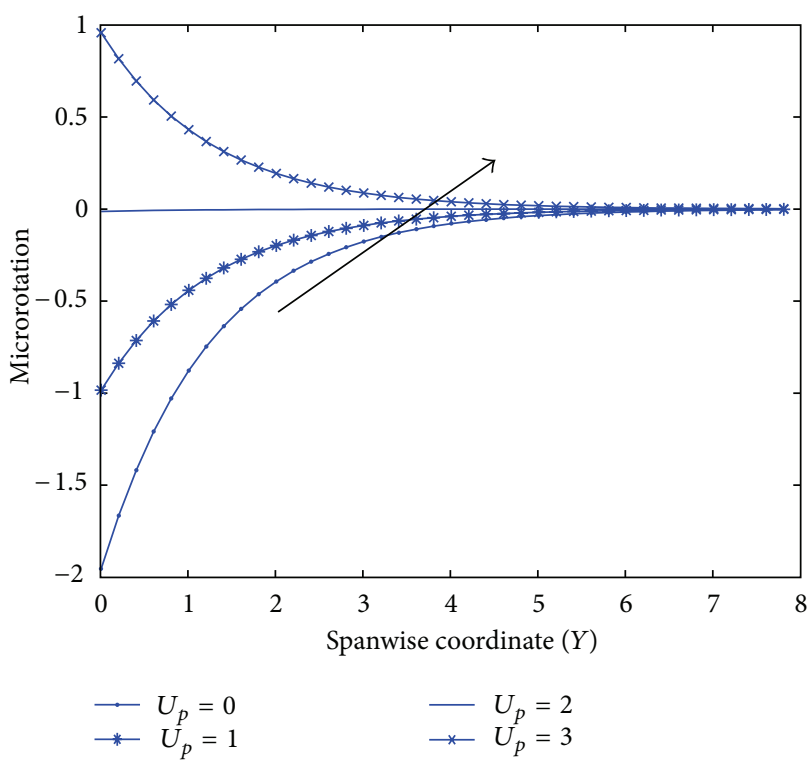

(b)

FIGURE 2: Velocity and microrotation distribution.

with an increase of $\zeta$, the translational velocity decreases, but the magnitude of microrotation increases as $\zeta$ decreases.

The effects of viscosity ratio $\beta$ on the translational velocity and the microrotation profiles across the boundary layer are presented in Figure 10. It is clear that the velocity distribution is greater for a Newtonian fluid $(\beta=0)$ with the fixed flow and material parameters, as compared with micropolar fluids. The results display that with an increase of viscosity ratio $\beta$, the translational velocity decreases, but the magnitude of microrotation increases as viscosity ratio $\beta$ increases. The translational velocity and the microrotation profiles against spanwise coordinate $y$ for different values of thermal Grashof number (Gr) are displayed in Figure 11. It is observed that an 


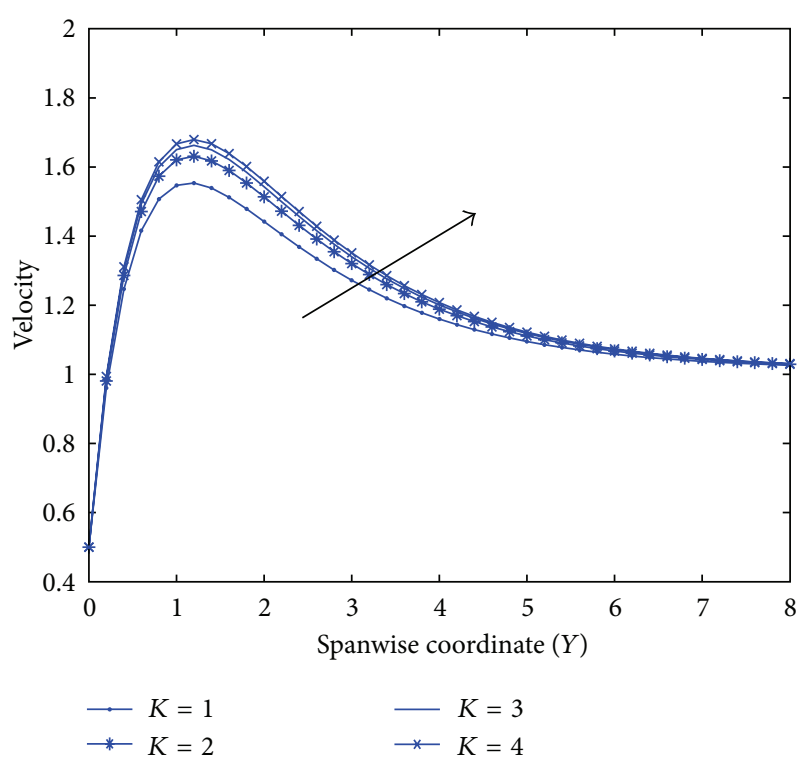

(a)

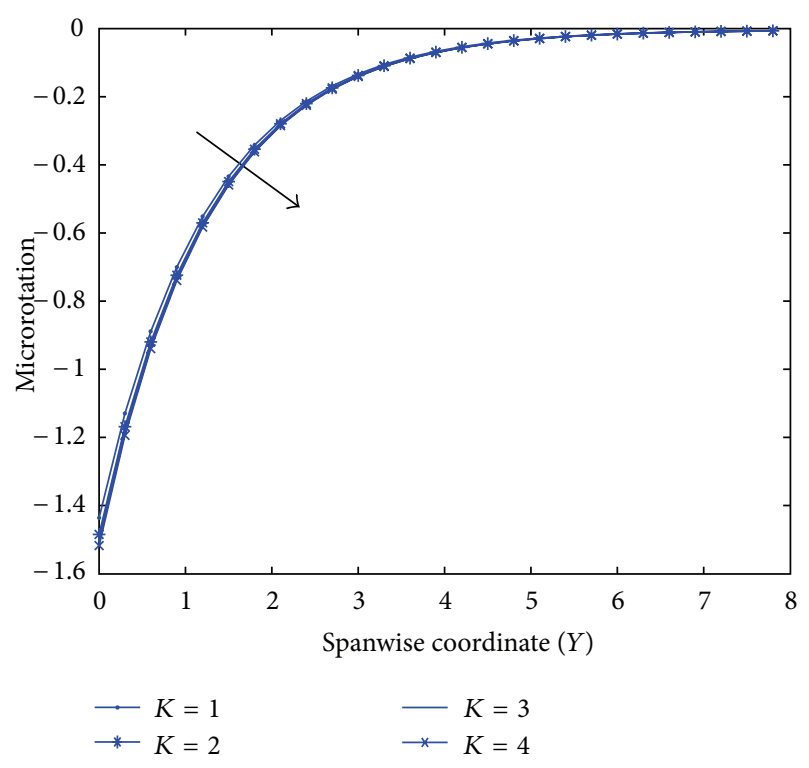

(b)

FIGURE 3: Velocity and microrotation distribution.

increase in Gr leads to an increase in the values of velocity and a decrease in the magnitude of microrotation. The translational velocity and the microrotation profiles against spanwise coordinate $y$ for different values of solutal Grashof number (Gc) are displayed in Figure 12. It is observed that an increase in $\mathrm{Gc}$ leads to an increase in the values of velocity and a decrease in the magnitude of microrotation.

Figure 13 shows the effect of $n$-parameter, which is related to microrotation vector and shear stress, on the translational velocity and the microrotation profiles. It is observed that the magnitude of velocity and microrotation increases with an

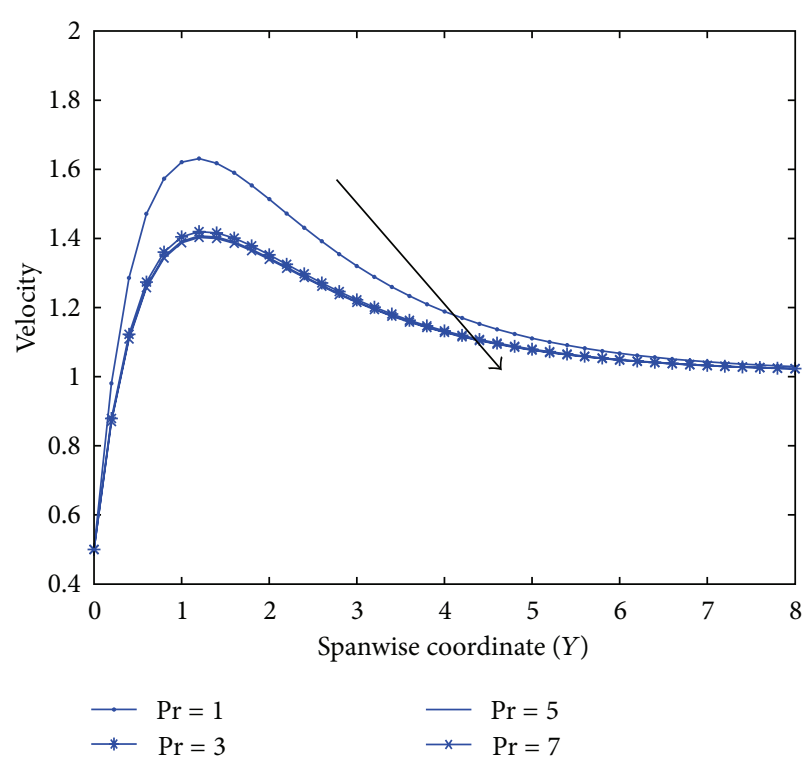

(a)

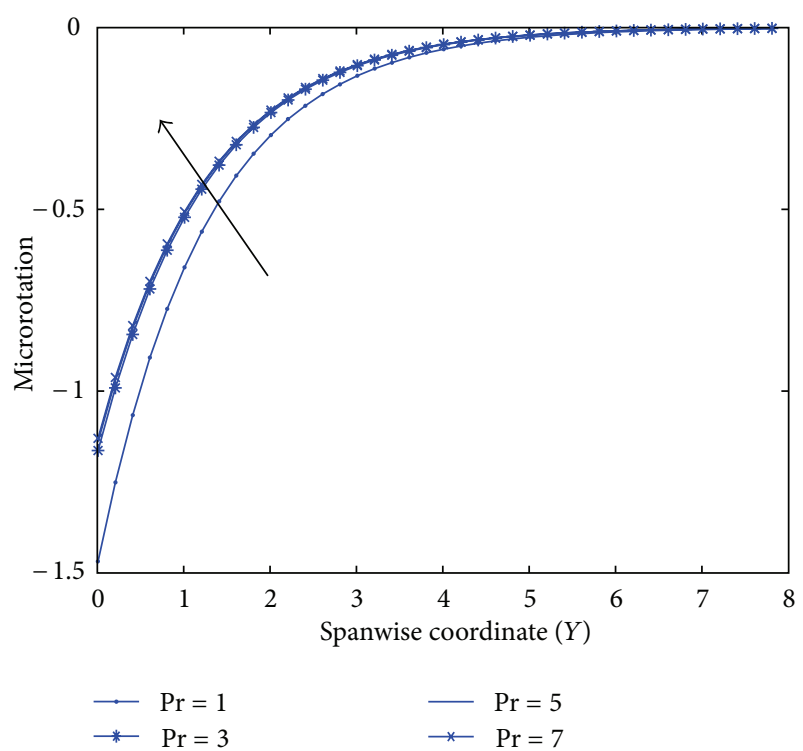

(b)

FigURE 4: Velocity and microrotation distribution.

increase of $n$-parameter, but the magnitude of microrotation decreases with an increase of $n$-parameter.

Figures 14(a) and 14(b) represent the temperature profiles for different values of thermal radiation parameter $R$ and prandtl number $(\mathrm{Pr})$ in the boundary layer. These figures indicate that the effect of thermal radiation is to enhance heat transfer because of the fact that thermal boundary layer thickness increases with an increase in the thermal radiation. Thus, it is pointed out that the radiation should be minimized to have the cooling process at a faster rate. But the temperature decreases with increasing the values of Prandtl number (Pr) in the boundary layer. From this plot, it is evident that the temperature in the boundary layer falls 


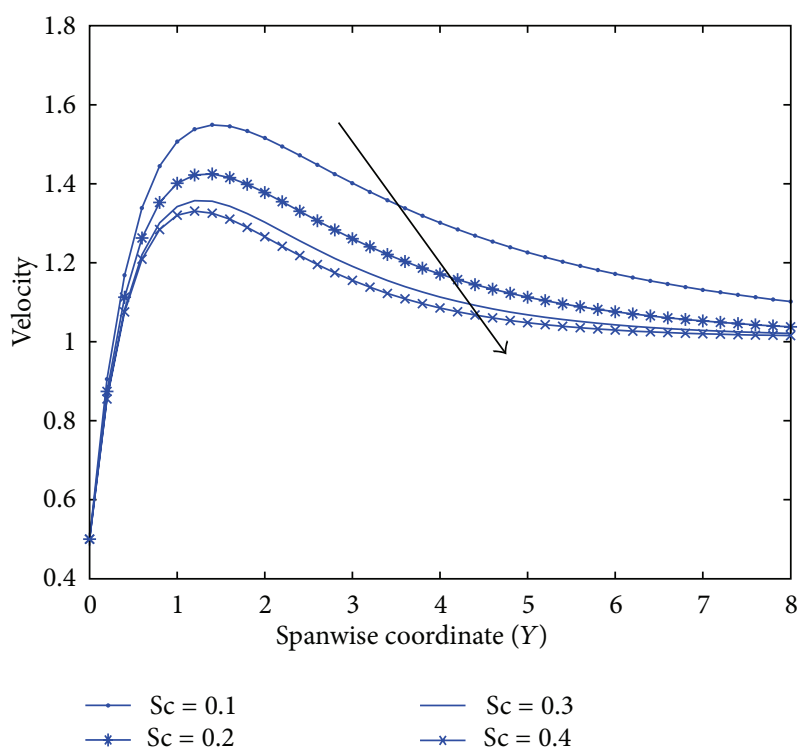

(a)

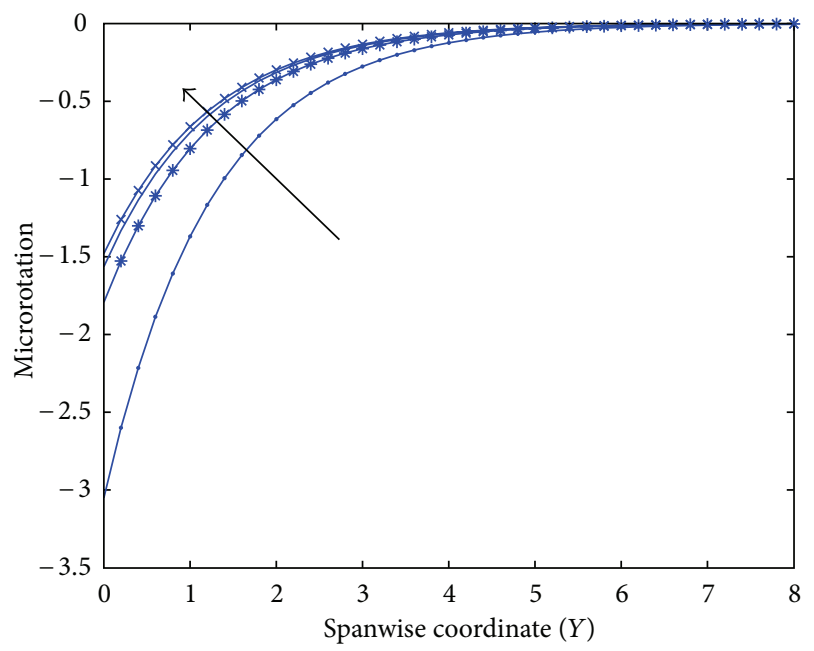

$\begin{array}{ll}\longrightarrow \mathrm{Sc}=0.1 & -\mathrm{Sc}=0.3 \\ \text { * } \mathrm{Sc}=0.2 & \rightarrow \mathrm{Sc}=0.4\end{array}$

(b)

FIGURE 5: Velocity and microrotation distribution.

very quickly for large value of the Prandtl number because of the fact that thickness of the boundary layer decreases with an increase in the value of the Prandtl number.

Typical variations of the temperature profiles along the spanwise coordinate $y$ are shown in Figure 14(c) for various values of $Q_{l}$. The results show that the temperature profiles decrease with increasing $Q_{l}$. The temperature profiles with respect to spanwise coordinate $y$ for various values of $\zeta$ are shown in Figure 14(d). The results show that as the chemical reaction parameter $\zeta$ increase, the thermal boundary layer increases.

The effect of the reaction rate parameter $\zeta$ on the species concentration profiles for generative chemical reaction is shown in Figure 15(a). It is noticed from the graph that there

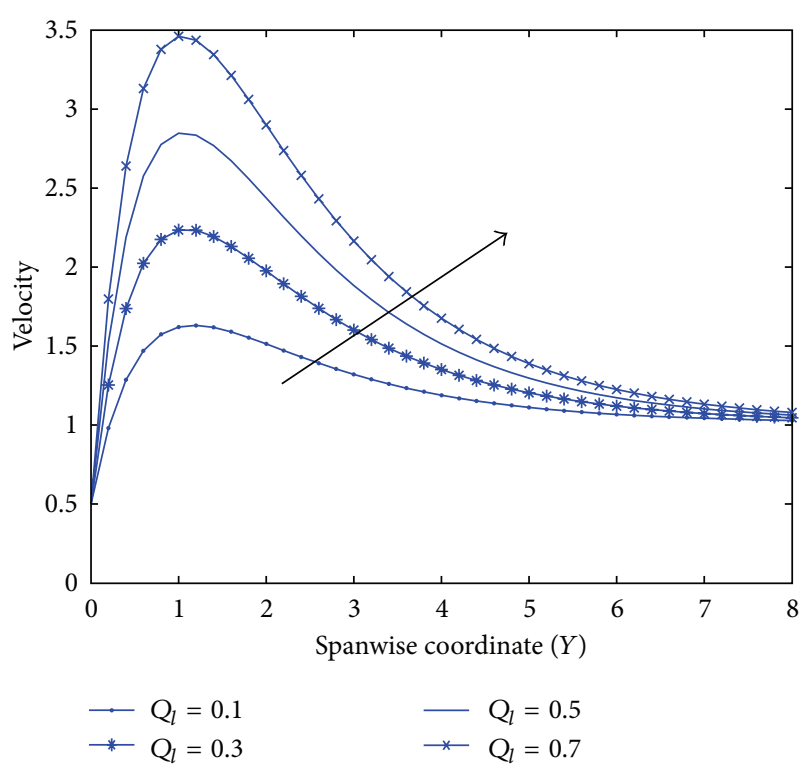

(a)

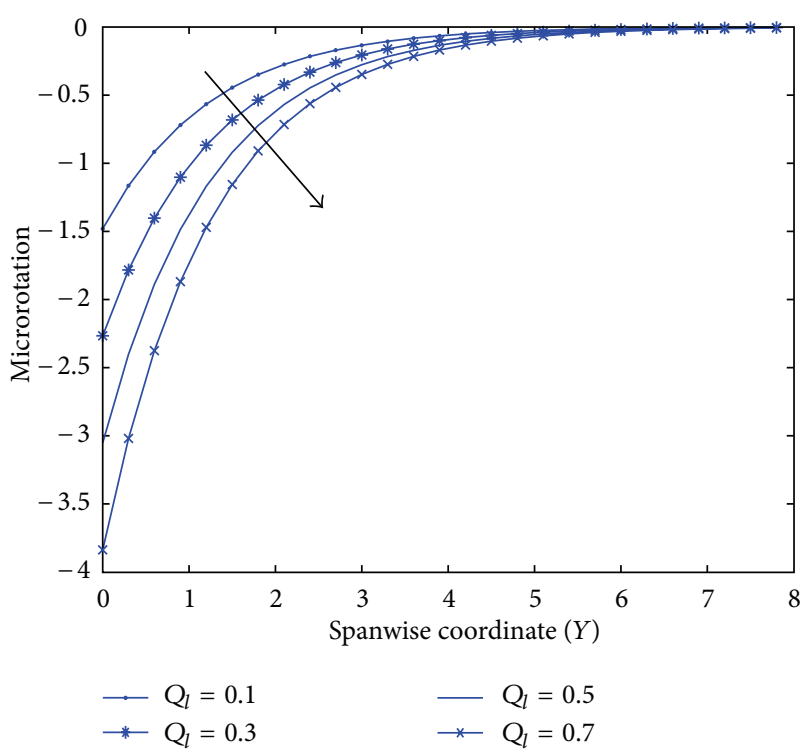

(b)

FIgURE 6: Velocity and microrotation distribution.

is a marked effect with increasing the value of the chemical reaction rate parameter $\zeta$ on the concentration distribution in the boundary layer. It is clearly observed from this figure that the concentration of species value of 1.0 at vertical plate decreases till it attains the minimum value of zero at the end of the boundary layer, and this trend is seen for all the values of reaction rate parameter. Further, it is observed that increasing the value of the chemical reaction decreases the concentration of species in the boundary layer; this is due to the fact that boundary layer decreases with increase in the value of $\zeta$ chemical reaction in this system result in the consumption of the chemical and hence result in decreasing concentration profile. Similar facts are seen in the case when 


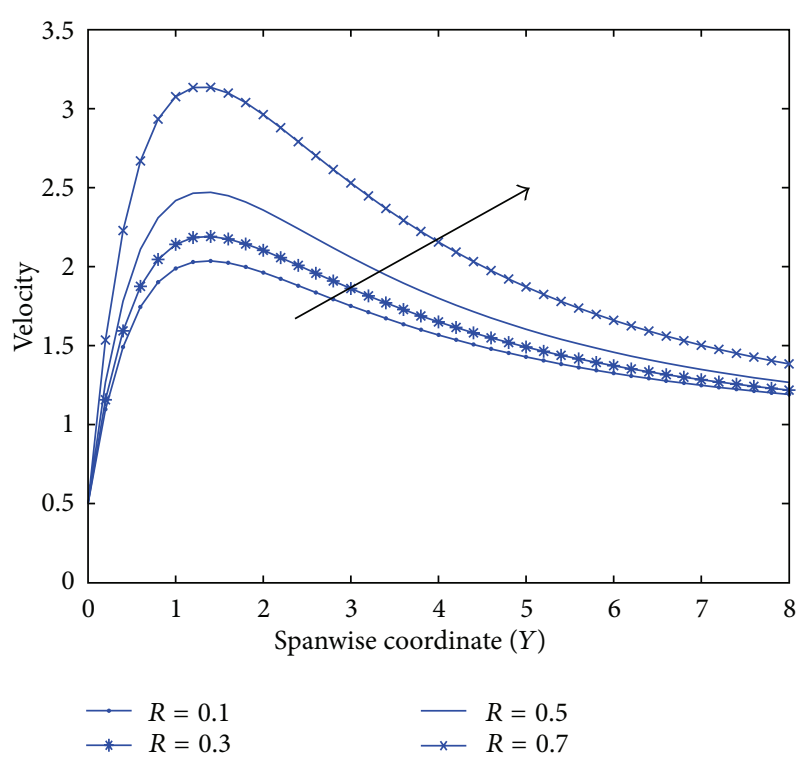

(a)

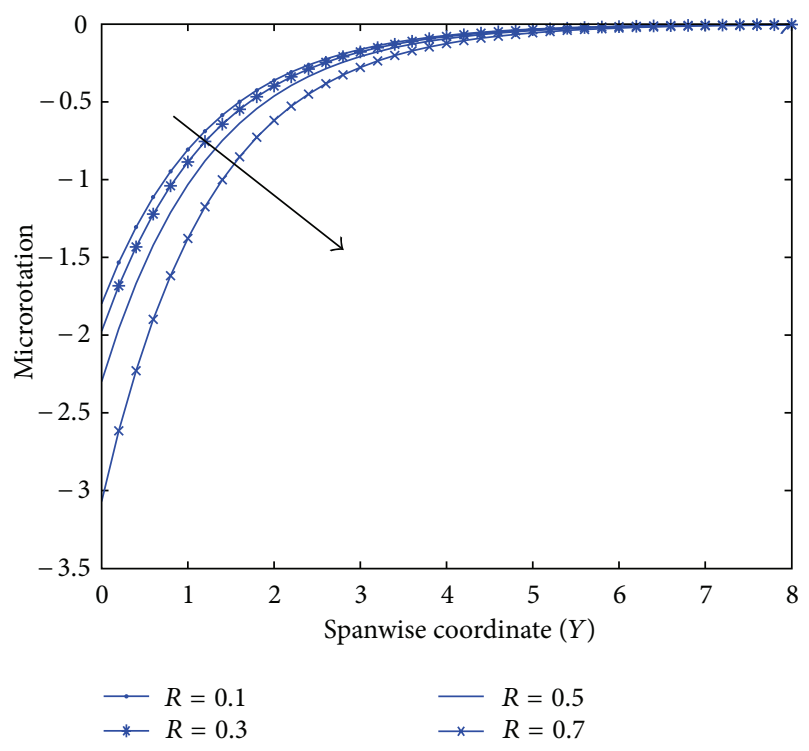

(b)

FIGURE 7: Velocity and microrotation distribution.

Schmidt number is increased as noted in Figure 15(b). It may also be observed from this figure that the effect of Schmidt number $(\mathrm{Sc})$ on concentration distribution decreases slowly for higher values of Sc. It is expected that the mass transfer rate increases as $\zeta$ increases with all other parameters fixed; that is, an increase in the Schmidt number $(\mathrm{Sc})$ produces a decrease in the concentration boundary layer thickness, associated with the reduction in the concentration profiles. Physically, the increase in the value of Sc means the decrease of molecular diffusion $D$. Hence, the concentration of the species is higher for smaller values of Sc and lower for larger values of Sc.

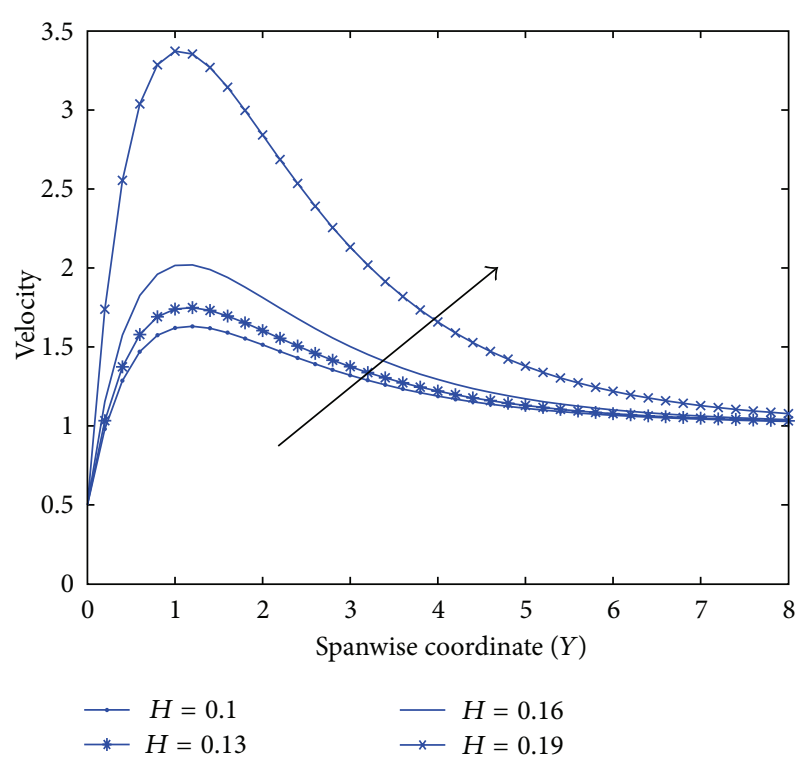

(a)

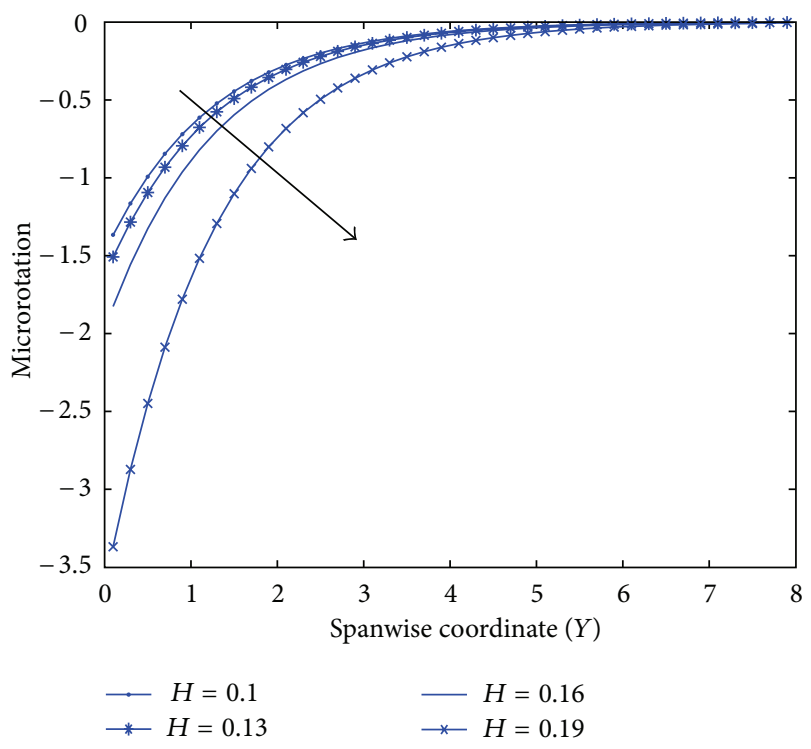

(b)

FIGURE 8: Velocity and Microrotation distribution.

\section{Conclusions}

The following conclusions are drawn from the present study.

(1) The effect of increasing magnetic parameter $M$ is to retard the transient velocity of the flow field at all points, while a growing Grashof number (Gr) for heat transfer or heat absorption parameter accelerates the transient velocity of the flow field at all points.

(2) A growing magnetic parameter $M$ or Prandtl number $(\mathrm{Pr})$ decelerates the transient temperature of the flow field at all points, while a growing permeability parameter $K$ or heat source parameter $S$ reverses the effect. 


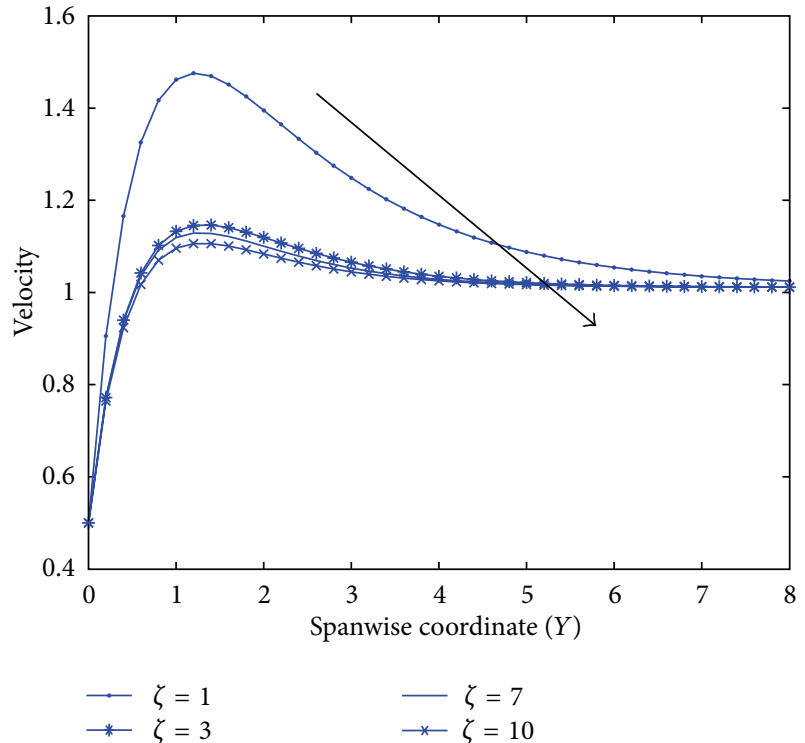

(a)

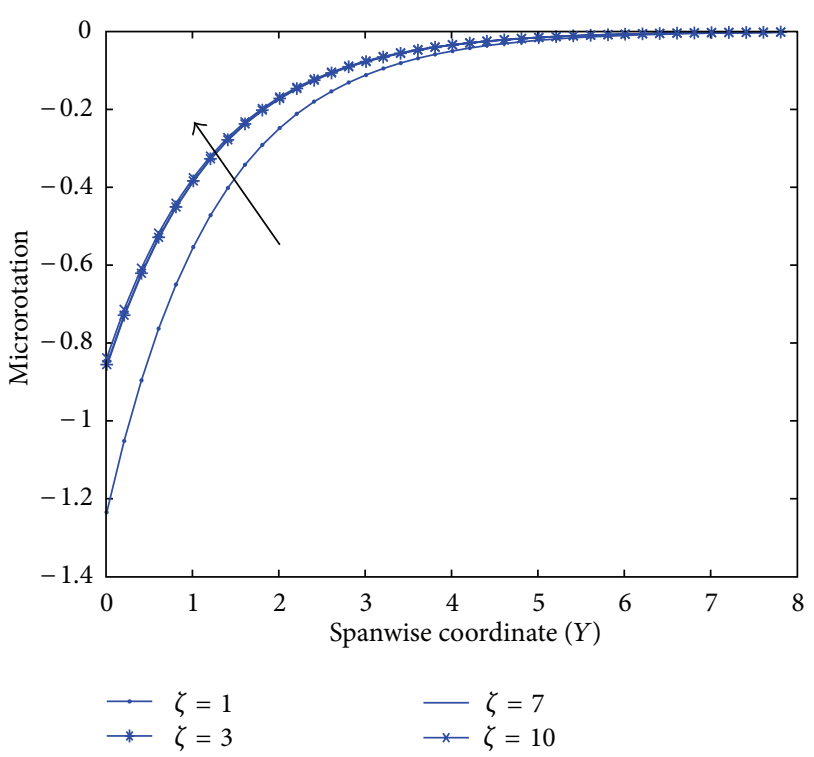

(b)

FIGURE 9: Velocity and microrotation distribution.

(3) The effect of increasing magnetic parameter $M$ is to reduce the skin-friction at the wall, while a growing permeability parameter $K$ or heat source parameter $S$ reverses the effect.

(4) A growing Prandtl number (Pr) or magnetic parameter $M$ increases the magnitude of the rate of heat transfer at the wall. On the other hand, a growing heat source $S$ parameter reverses the effect.

(5) Skin-friction decreases when, magnetic field parameter is increased.

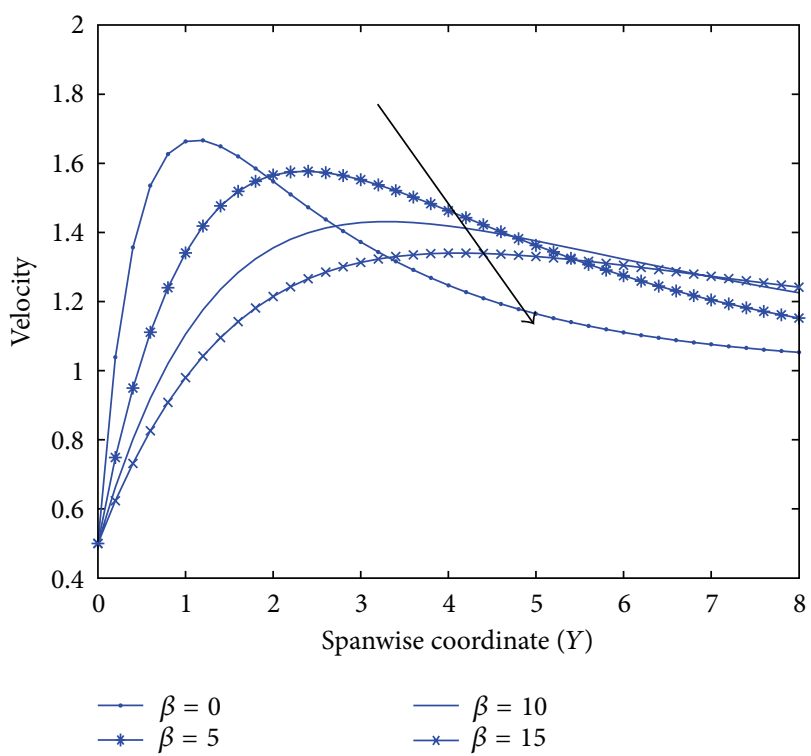

(a)

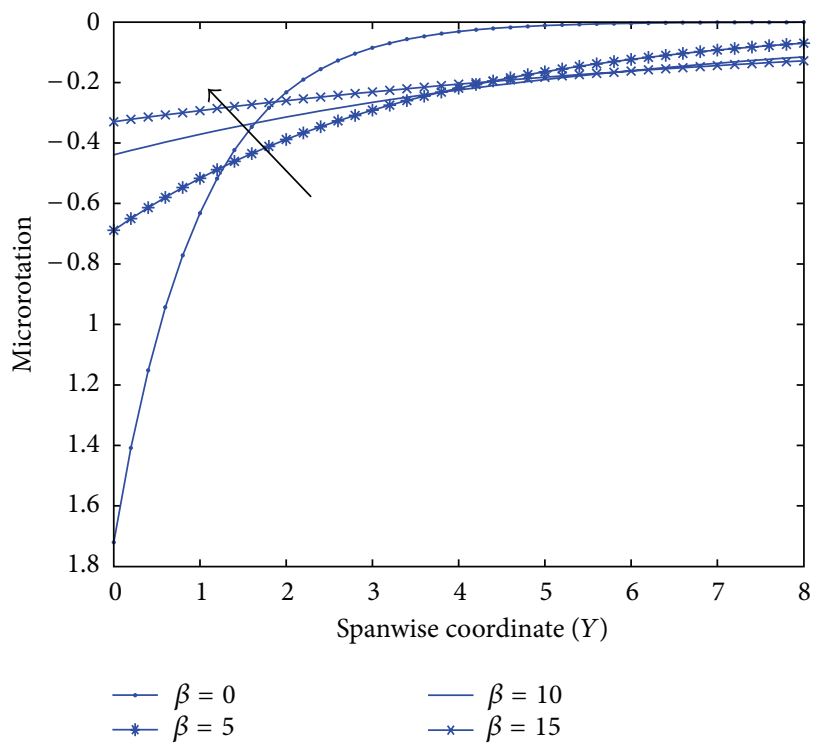

(b)

FIGURE 10: Velocity and microrotation distribution.

\section{Appendix}

Consider

$$
\begin{gathered}
h_{1}=\frac{s_{c}}{2}\left[1+\sqrt{1+\frac{4 \zeta}{s_{c}}}\right], \\
h_{2}=\frac{s_{c}}{2}\left[1+\sqrt{1+\frac{4(\delta+\zeta)}{s_{c}}}\right], \\
h_{3}=\frac{3 \operatorname{Pr}}{2(3+4 R)}\left[1+\sqrt{1-\frac{4 H(3+4 R)}{3 \operatorname{Pr}}}\right],
\end{gathered}
$$




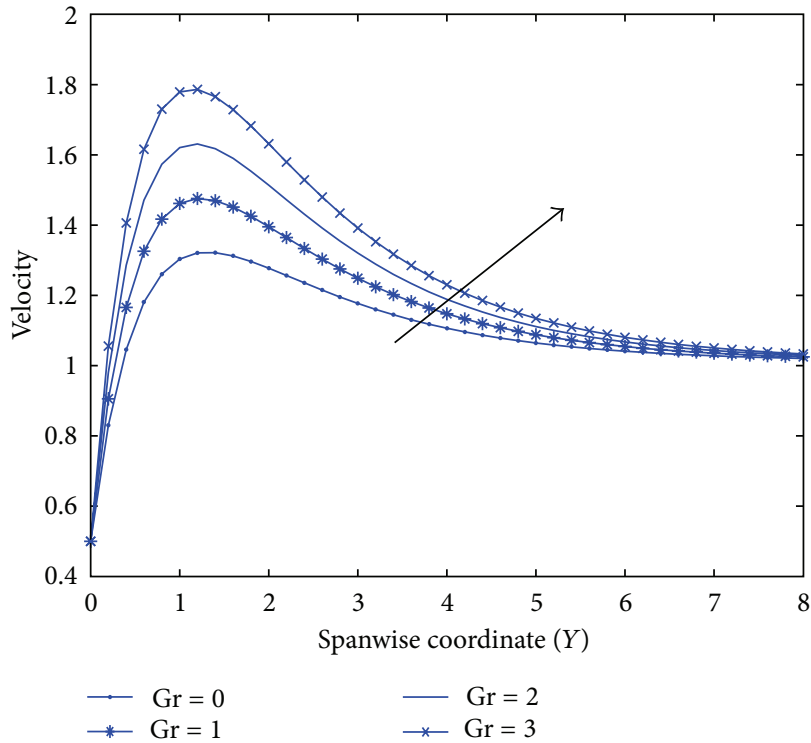

(a)

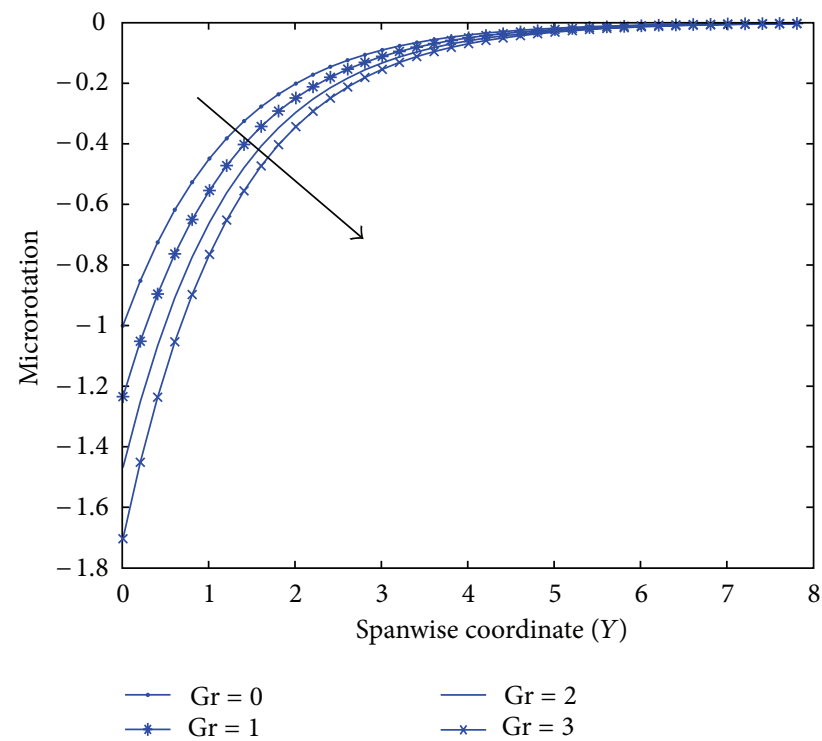

(b)

FIGURE 11: Velocity and microrotation distribution.

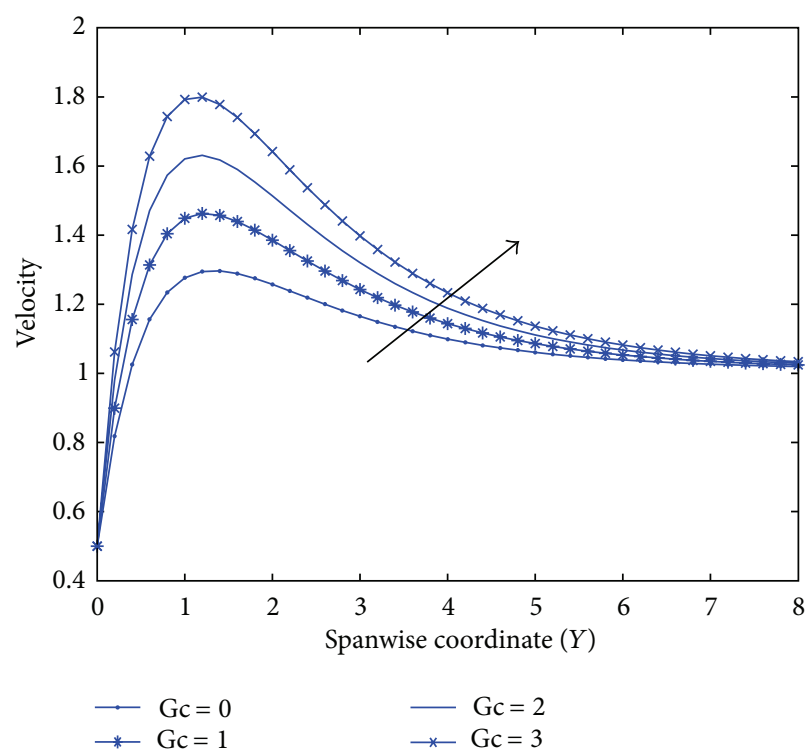

(a)

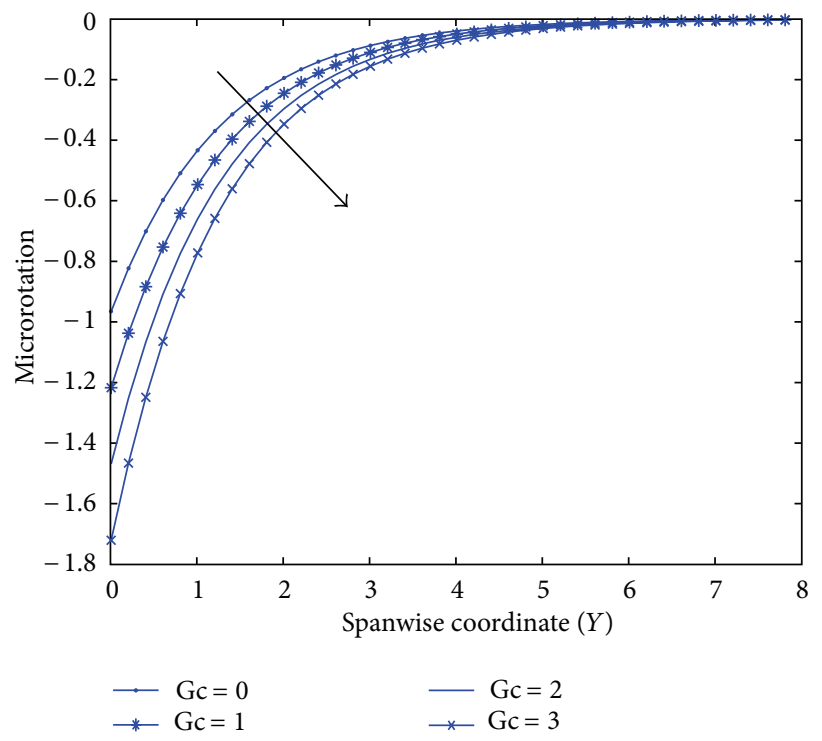

(b)

FIGURE 12: Velocity and microrotation distribution.

$$
\begin{array}{cc}
h_{4}=\frac{3 \operatorname{Pr}}{2(3+4 R)}\left[1+\sqrt{1+\frac{4(\delta-H)(3+4 R)}{3 \operatorname{Pr}}}\right], & R_{3}=\frac{1}{2(1+\beta)}[1+\sqrt{1+4(N+\delta)(1+\beta)}] \\
h_{6}=\frac{\eta}{2}\left[1+\sqrt{1+\frac{(4 \delta)}{\eta}}\right], & z_{2}=\frac{A s_{c} h_{1}}{h_{1}^{2}-h_{1} s_{c}-(\delta+\zeta) s_{c}}, \\
R_{1}=\frac{1}{2(1+\beta)}[1+\sqrt{1+(N+\delta)(1+\beta)}], & z_{3}=\frac{-3 \operatorname{Pr} Q_{l}}{(3+4 R) h_{1}^{2}-3 \operatorname{Pr} h_{1}+3 \operatorname{Pr} H},
\end{array}
$$




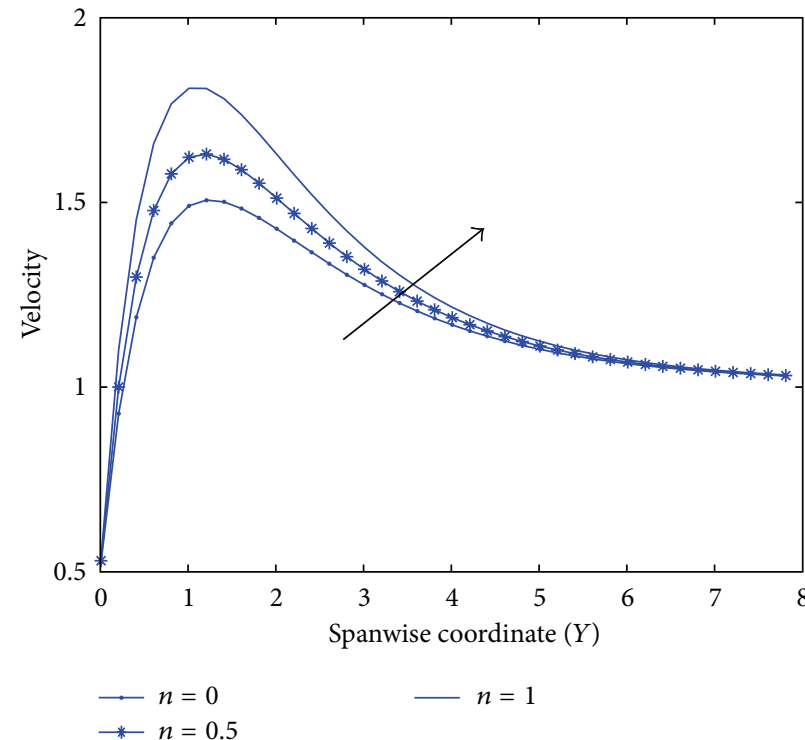

(a)

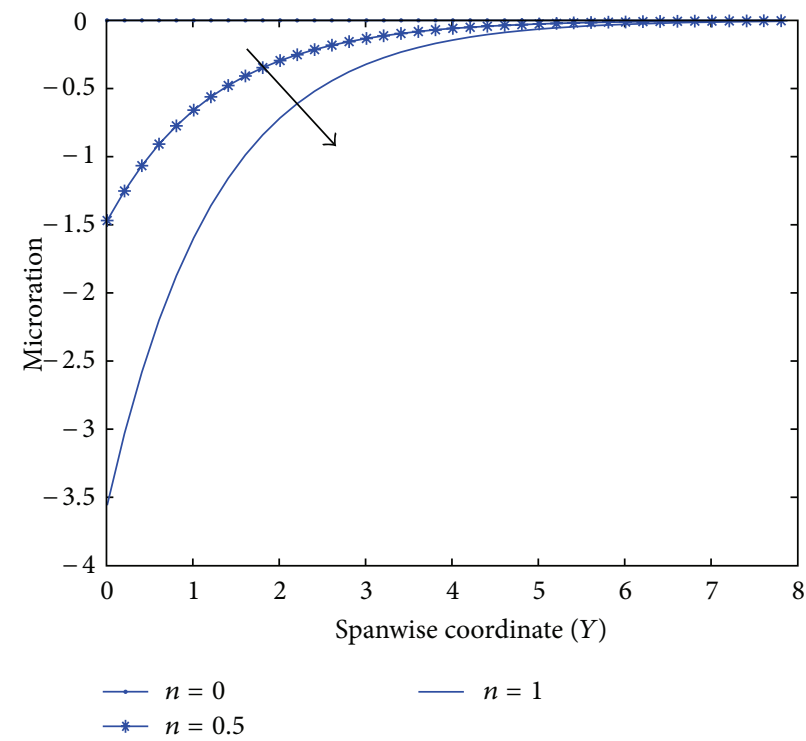

(b)

FIGURE 13: Velocity and microrotation distribution.

$$
\begin{aligned}
& z_{4}=\frac{3 \operatorname{Pr}_{3}\left(1-z_{3}\right)}{(3+4 R) h_{3}^{2}-3 \operatorname{Pr} h_{3}-3 \operatorname{Pr}(\delta-H)}, \\
& z_{5}=\frac{3 \operatorname{Pr} h_{1} z_{3}}{(3+4 R) h_{1}^{2}-3 \operatorname{Pr} h_{1}-3 \operatorname{Pr}(\delta-H)}, \\
& z_{6}=\frac{-3 \operatorname{PrQ}_{l}\left(1-z_{2}\right)}{(3+4 R) h_{2}^{2}-3 \operatorname{Pr} h_{2}-3 \operatorname{Pr}(\delta-H)}, \\
& z_{7}=\frac{-3 \operatorname{PrQ} z_{l} z_{2}}{(3+4 R) h_{1}^{2}-3 \operatorname{Pr} h_{1}-3 \operatorname{Pr}(\delta-H)}, \\
& a_{2}=\frac{-\mathrm{Gr}\left(1-z_{3}\right)}{(1+\beta) h_{3}^{2}-h_{3}-N}, \\
& a_{3}=\frac{-\mathrm{Gr} z_{3}}{(1+\beta) h_{1}^{2}-h_{1}-N} \\
& a_{4}=\frac{-\mathrm{Gc}}{(1+\beta) h_{1}^{2}-h_{1}-N} \text {, } \\
& P=\frac{2 \beta \eta}{(1+\beta) \eta^{2}-\eta-N} \\
& a_{6}=a_{3}+a_{4} \\
& D=\frac{n}{1+n\left(R_{1}-\eta\right) P}, \\
& c_{11}=D\left[\left(U_{p}-1\right) R_{1}+a_{2}\left(h_{3}-R_{1}\right)+a_{6}\left(h_{1}-R_{1}\right)\right] \text {, } \\
& a_{5}=P^{*} c_{11} ; \quad a_{1}=U_{p}-1-a_{2}-a_{6}-a_{5}, \\
& z_{8}=z_{5}+z_{7} \\
& c_{9}=1-z_{4}-z_{6}-z_{8} \text {, }
\end{aligned}
$$$$
b_{2}=\frac{-\mathrm{Grc}_{9}}{(1+\beta) h_{4}^{2}-h_{4}-(N+\delta)},
$$$$
b_{3}=\frac{-\mathrm{Gr} z_{4}}{(1+\beta) h_{3}^{2}-h_{3}-(N+\delta)},
$$$$
b_{4}=\frac{-\mathrm{Gr} z_{6}}{(1+\beta) h_{2}^{2}-h_{2}-(N+\delta)},
$$$$
b_{5}=\frac{-\mathrm{Gr} z_{8}}{(1+\beta) h_{1}^{2}-h_{1}-(N+\delta)},
$$$$
b_{6}=\frac{-\mathrm{Gc}\left(1-z_{2}\right)}{(1+\beta) h_{2}^{2}-h_{2}-(N+\delta)},
$$$$
b_{7}=\frac{-\mathrm{Gc} z_{2}}{(1+\beta) h_{1}^{2}-h_{1}-(N+\delta)} \text {, }
$$$$
D_{1}=\frac{-2 \beta A \eta^{2} c_{11}}{\delta},
$$$$
b_{9}=\frac{D_{1}}{(1+\beta) \eta^{2}-\eta-(N-\delta)} \text {, }
$$$$
b_{10}=\frac{A R_{1} a_{1}}{(1+\beta) R_{1}^{2}-R_{1}-(N+\delta)} \text {, }
$$$$
b_{11}=\frac{A h_{3} a_{2}}{(1+\beta) h_{3}^{2}-h_{3}-(N+\delta)} \text {, }
$$$$
b_{12}=\frac{A h_{1} a_{6}}{(1+\beta) h_{1}^{2}-h_{1}-(N+\delta)} \text {, }
$$$$
b_{13}=\frac{A \eta a_{5}}{(1+\beta) \eta^{2}-\eta-(N+\delta)},
$$

$$
d_{1}=b_{3}+b_{11} \text {, }
$$




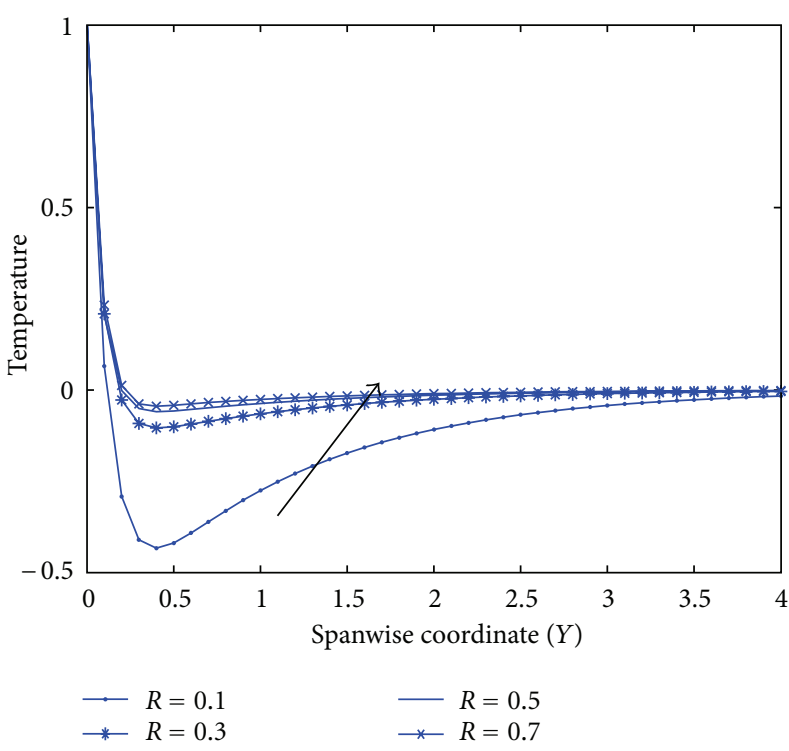

(a)

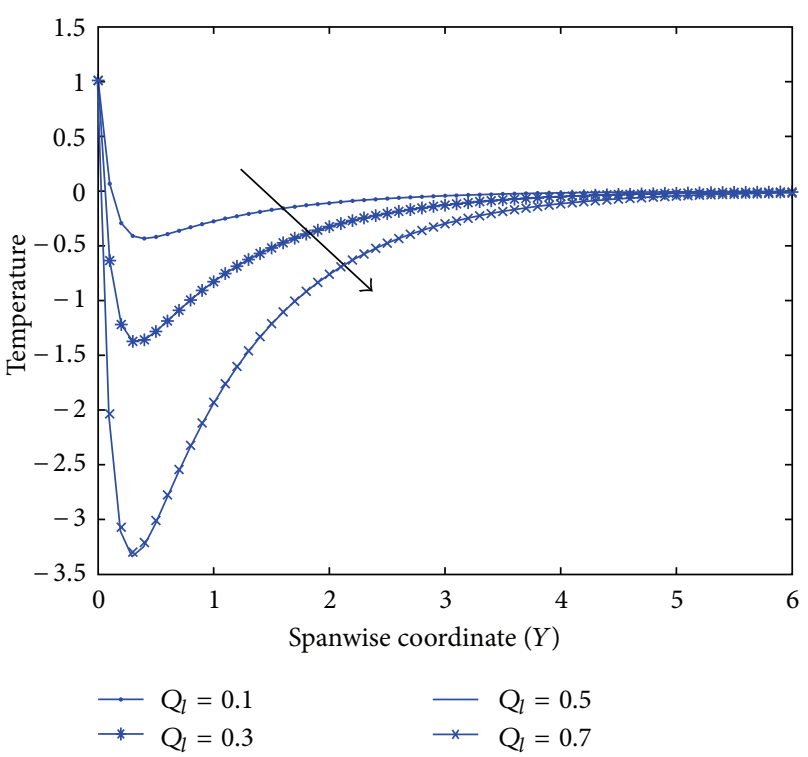

(c)

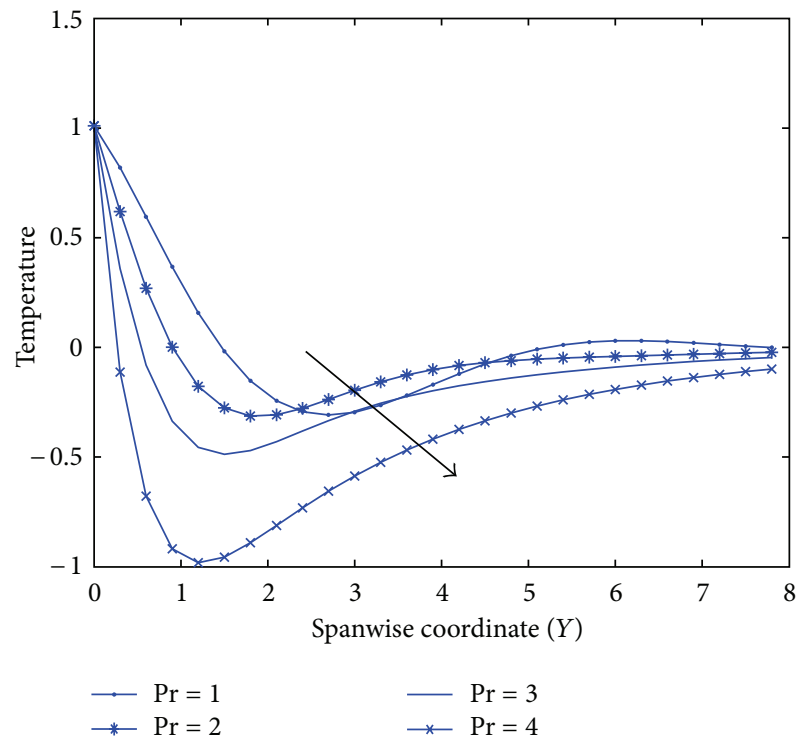

(b)

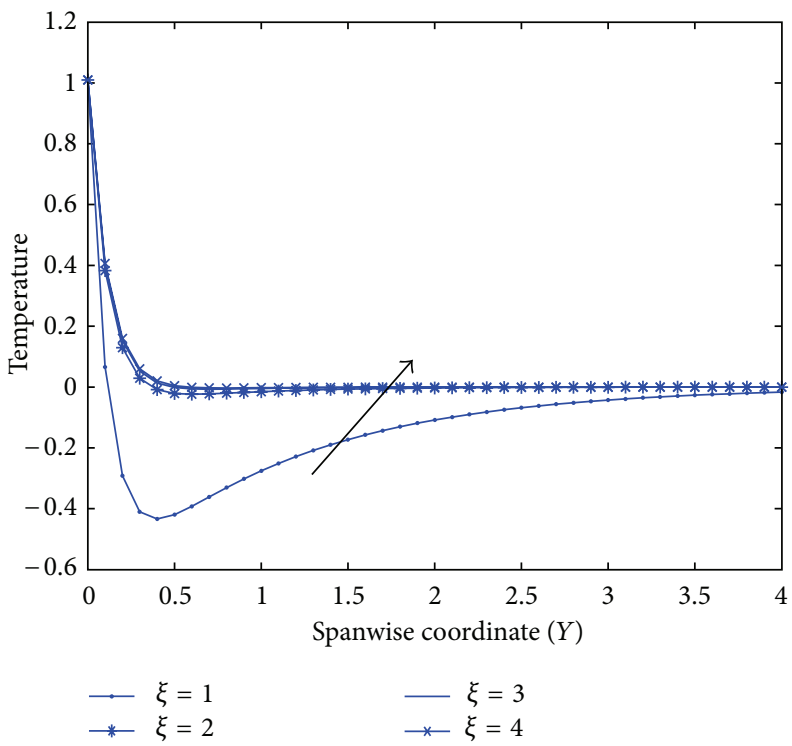

(d)

FIgURE 14: Temperature distribution.

$$
\begin{gathered}
d_{2}=b_{4}+b_{6}, \\
d_{3}=b_{5}+b_{7}+b_{12}, \\
d_{4}=b_{9}+b_{13}, \\
Q=\frac{2 \beta h_{6}}{(1+\beta) h_{6}^{2}-h_{6}-(N-\delta)}, \\
k_{1}=\frac{A \eta c_{11}}{\delta}+n\left[b_{2}\left(b_{4}-R_{3}\right)+d_{1}\left(h_{3}-R_{3}\right)\right. \\
+d_{2}\left(h_{2}-R_{3}\right)+d_{3}\left(h_{1}-R_{3}\right) \\
\left.+d_{4}\left(\eta-R_{3}\right)+b_{10}\left(R_{1}-R_{3}\right)-R_{3}\right],
\end{gathered}
$$

$$
\begin{gathered}
c_{13}=\frac{1}{1+n\left(R_{3}-h_{6}\right) Q} * \kappa_{1}, \\
b_{8}=Q c_{13}, \\
b_{1}=-\left(1+b_{2}+d_{1}+d_{2}+d_{3}+d_{4}+b_{8}+b_{10}\right) .
\end{gathered}
$$

\section{Nomenclature}

$M$ : The local magnetic field parameter

$n$ : The parameter related to microrotation vector and shear stress

$N$ : The model parameter 


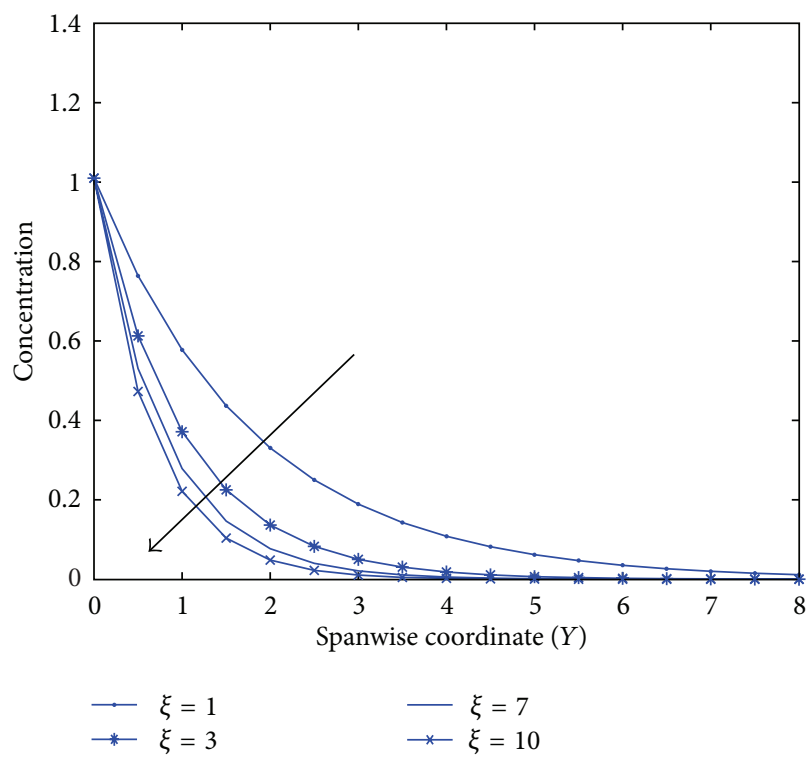

(a)

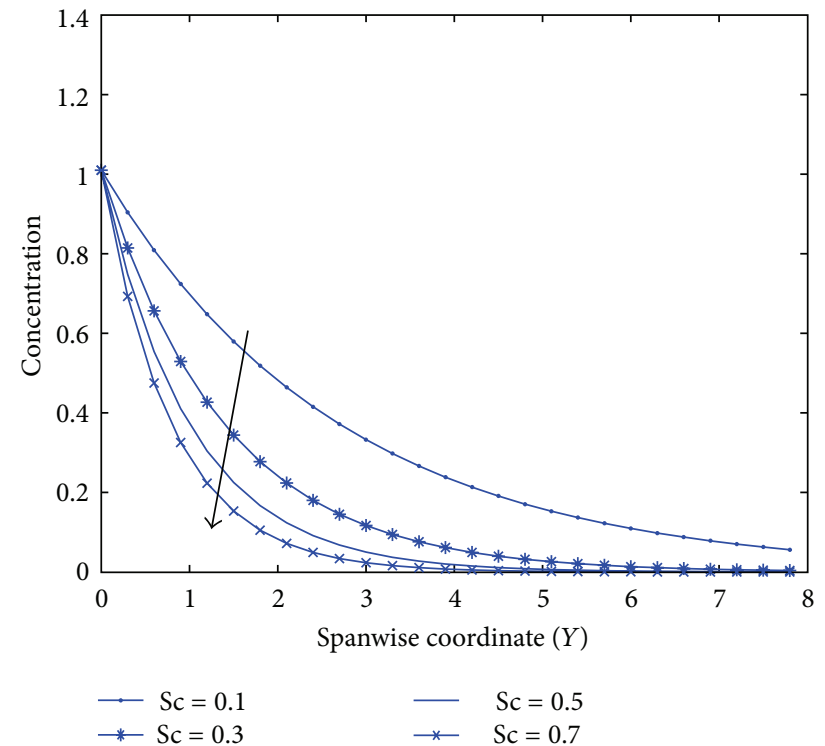

(b)

FIGURE 15: Concentration distribution.

\begin{tabular}{|c|c|}
\hline $\mathrm{Nu}:$ & Nusselt number \\
\hline$P^{*}:$ & The pressure, $\mathrm{kg} \mathrm{m}^{-1} \mathrm{~s}^{-2}$ \\
\hline Pr: & The fluid Prandtl number \\
\hline$Q_{0}:$ & The heat generation coefficient, $\mathrm{W} \mathrm{m}{ }^{-3} \mathrm{~K}^{-1}$ \\
\hline$q_{r}^{*}:$ & The radiative heat flux, $\mathrm{W} \mathrm{m}^{-2}$ \\
\hline$R^{*}:$ & The reaction rate constant, $\mathrm{J}$ \\
\hline$R:$ & The radiation parameter \\
\hline Re: & The Reynolds number \\
\hline Sc: & The generalized Schmidt number \\
\hline Sh: & Sherwood number \\
\hline$Q_{l}:$ & The radiation absorption number \\
\hline$t^{*}:$ & The time, $s$ \\
\hline$t:$ & The dimensionless time \\
\hline$T^{*}:$ & The temperature in the boundary layer, $\mathrm{K}$ \\
\hline$T_{\infty}^{*}:$ & The temperature far away from the plate, $\mathrm{K}$ \\
\hline$T_{w}^{*}:$ & The temperature at the wall, $\mathrm{K}$ \\
\hline$u:$ & The dimensionless velocity \\
\hline$u^{*}, v^{*}:$ & $\begin{array}{l}\text { The velocities along and perpendicular to the } \\
\text { plate, } \mathrm{m} \mathrm{s}^{-1}\end{array}$ \\
\hline$U_{0}:$ & The scale of free stream velocity \\
\hline$U_{\infty}^{*}:$ & The free stream velocity, $\mathrm{m} \mathrm{s}^{-1}$ \\
\hline$u_{p}^{*}:$ & The wall dimensional velocity, $\mathrm{m} \mathrm{s}^{-1}$ \\
\hline$U_{p}^{P}:$ & The wall dimensionless velocity \\
\hline$V_{0}:$ & The scale of suction velocity \\
\hline$x^{*}$ and $y^{*}$ & $\begin{array}{l}\text { The distances along and perpendicular to the } \\
\text { plate, respectively, } m\end{array}$ \\
\hline$y:$ & $\begin{array}{l}\text { The dimensionless spanwise coordinate } \\
\text { normal to the plate }\end{array}$ \\
\hline$\gamma:$ & The spingradient velocity, $\mathrm{kg} \mathrm{m} \mathrm{s}^{-1}$ \\
\hline$\delta:$ & The scalar constant \\
\hline$\varepsilon:$ & The scalar constant \\
\hline$\theta:$ & The dimensionless temperature \\
\hline$\zeta:$ & The chemical reaction parameter \\
\hline$\eta:$ & The scalar constant \\
\hline
\end{tabular}

$\mu: \quad$ The fluid dynamic viscosity, $\mathrm{kg} \mathrm{m}^{-1} \mathrm{~s}^{-1}$

$v$ : The fluid kinematic rotational viscosity, $\mathrm{m}^{2} \mathrm{~s}^{-1}$

$v_{r}$ : The kinematic rotational viscosity, $\mathrm{m}^{2} \mathrm{~s}^{-1}$

$\rho: \quad$ Fluid density, $\mathrm{kg} \mathrm{m}^{-3}$

$\sigma: \quad$ The electrical conductivity of the fluid, $\mathrm{S} \mathrm{m}^{-1}$

$\sigma^{*}$ : The Stefan-Boltzmann constant, $\mathrm{W} \mathrm{m}^{-2} \mathrm{~K}^{-4}$

$\omega$ : Angular velocity vector, $\mathrm{m} \mathrm{s}^{-1}$

$\Gamma$ : $\quad$ The coefficient of gyro-viscosity (or vortex viscosity), $\mathrm{kg} \mathrm{m}^{-1} \mathrm{~s}^{-1}$

$B_{0}$ : The magnetic induction, tesla

$C^{*}$ : The species concentration, $\mathrm{mol} \mathrm{m}^{-3}$

$C_{\omega}^{*}$ : The surface concentration, $\mathrm{mol} \mathrm{m}^{-3}$

$C_{\infty}^{*}$ : The species concentration far from the surface, $\mathrm{mol} \mathrm{m}^{-3}$

$C_{f}$ : The skin-friction coefficient

$C_{m}$ : The couple stress coefficient

$C_{p}$ : The specific heat at constant pressure, $\mathrm{J} \mathrm{kg}^{-1} \mathrm{~K}^{-1}$

$D^{*}$ : The chemical molecular diffusivity, $\mathrm{m}^{2} \mathrm{~s}^{-1}$

Gm: The solutal Grashof number

Gr: Grashof number

g: The acceleration due to the gravity, $\mathrm{m} \mathrm{s}^{-2}$

$H$ : The dimensionless heat generation/absorption coefficient

$j^{*}: \quad$ The microinertia per unit mass, $\mathrm{m}^{2}$

K: $\quad$ The permeability of the porous media, $\mathrm{m}^{2}$

$K_{1}^{*}$ : The mean absorption coefficient, $\mathrm{m}^{-1}$

$K$ : The dimensionless permeability

$k$ : The thermal conductivity of the fluid, $\mathrm{W} \mathrm{m}^{-1} \mathrm{~K}^{-1}$

$\alpha$ : The fluid thermal diffusivity, $\mathrm{m}^{2} \mathrm{~s}^{-1}$

$\beta$ : The ratio of vortex viscosity and dynamic viscosity 
$\beta_{c}$ : Volumetric coefficient of thermal expansion, $\mathrm{K}^{-1}$

$\beta_{f}$ : Volumetric coefficient of expansion with concentration, $\mathrm{K}^{-1}$

$B$ : The scalar constant.

\section{References}

[1] A. C. Eringen, "Simple microfluids," International Journal of Engineering Science, vol. 2, pp. 205-217, 1964.

[2] A. C. Eringen, "Theory of termomicrofluids," Journal of Mathematical Analysis and Applications, vol. 38, pp. 480-496, 1972.

[3] T. Ariman, M. A. Turk, and N. D. Sylvester, "Microcontinuum fluid mechanics: a review," International Journal of Engineering Science, vol. 11, no. 8, pp. 905-930, 1973.

[4] R. S. R. Gorla, "Mixed convection in a micropolar fluid from a vertical surface with uniform heat flux," International Journal of Engineering Science, vol. 30, no. 3, pp. 349-358, 1992.

[5] Y. J. Kim, "Unsteady convetion flow of micropolar fluids past a vertical porous plate embedded in a porous medium," Acta Mechanica, vol. 148, no. 1-4, pp. 105-116, 2001.

[6] G. R. Charya, "Flow of micropolar fluid through a constricted channel," International Journal of Engineering Science, vol. 15, no. 12, pp. 719-725, 1977.

[7] D. A. S. Rees and I. Pop, "Free convection boundary-layer flow of a micropolar fluid from a vertical flat plate," IMA Journal of Applied Mathematics, vol. 61, no. 2, pp. 179-197, 1998.

[8] R. C. Sharma and U. Gupta, "Thermal convection in micropolar fluids in porous medium," International Journal of Engineering Science, vol. 33, no. 13, pp. 1887-1892, 1995.

[9] J. P. Kumar, J. C. Umavathi, A. J. Chamkha, and I. Pop, "Fullydeveloped free-convective flow of micropolar and viscous fluids in a vertical channel," Applied Mathematical Modelling, vol. 34, no. 5, pp. 1175-1186, 2010.

[10] P. Muthu, B. V. Rathish Kumar, and P. Chandra, "Peristaltic motion of micropolar fluid in circular cylindrical tubes: effect of wall properties," Applied Mathematical Modelling, vol. 32, no. 10, pp. 2019-2033, 2008.

[11] D. Srinivasacharya, J. V. Ramana Murthy, and D. Venugopalam, "Unsteady stokes flow of micropolar fluid between two parallel porous plates," International Journal of Engineering Science, vol. 39, no. 14, pp. 1557-1563, 2001.

[12] R. Muthuraj and S. Srinivas, "Fully developed mhd flow of a micropolar and viscous fluids in a vertical porous space using HAM," Journal of Applied Mathematics and Mechanics, vol. 6, no. 11, pp. 55-78, 2010.

[13] U. N. Das, R. Deka, and V. M. Soundalgekar, "Effects of mass transfer on flow past an impulsively started infinite vertical plate with constant heat flux and chemical reaction," Forschung im Ingenieurwesen Engineering Research, vol. 60, no. 10, pp. 284287, 1994.

[14] R. Muthucumaraswamy and P. Ganesan, "First-order chemical reaction on flow past an impulsively started vertical plate with uniform heat and mass flux," Acta Mechanica, vol. 147, no. 1-4, pp. 45-57, 2001.

[15] P. V. Satya Narayana, D. Ch. Kesavaiah, and S. Venkataramana, "Viscous dissipation and thermal radiation effects on an unsteady MHD convection flow past a semi-infinite vertical permeable moving porous plate," International Journal of Mathematical Archive, vol. 2, no. 4, pp. 476-487, 2011.
[16] R. Kandasamy, K. Periasamy, and K. K. S. Prabhu, "Effects of chemical reaction, heat and mass transfer along a wedge with heat source and concentration in the presence of suction or injection," International Journal of Heat and Mass Transfer, vol. 48, no. 7, pp. 1388-1394, 2005.

[17] J. Gireesh Kumar, P. V. Satya Narayana, and S. Ramakrishna, "Effects of the chemical reaction and mass transfer on MHD unsteady free convection flow past an infinite vertical plate with constant suction and heat sink," Ultra Science, vol. 21, no. 3, pp. 639-650, 2009.

[18] G. R. Reddy, P. V. S. Narayana, and S. Venkataramana, "Peristaltic transport of a conducting fluid in an inclined asymmetric channel," Applied Mathematical Sciences, vol. 4, no. 35, pp. 1729$1741,2010$.

[19] D. Pal and B. Talukdar, "Perturbation analysis of unsteady magnetohydrodynamic convective heat and mass transfer in a boundary layer slip flow past a vertical permeable plate with thermal radiation and chemical reaction," Communications in Nonlinear Science and Numerical Simulation, vol. 15, no. 7, pp. 1813-1830, 2010.

[20] E. M. Abo-Eldahab and M. A. El Aziz, "Flow and heat transfer in a micropolar fluid past a stretching surface embedded in a nonDarcian porous medium with uniform free stream," Applied Mathematics and Computation, vol. 162, no. 2, pp. 881-899, 2005.

[21] R. S. R. Gorla, A. A. Mohammedien, M. A. Mansour, and I. A. Hassanien, "Unsteady natural convection from a heated vertical plate in micropolar fluid," Numerical Heat Transfer A, vol. 28, no. 2, pp. 253-262, 1995.

[22] A. Raptis and C. Perdikis, "Viscous flow over a non-linearly stretching sheet in the presence of a chemical reaction and magnetic field," International Journal of Non-Linear Mechanics, vol. 41, no. 4, pp. 527-529, 2006.

[23] D. Ch. Kesavaiah, P. V. Satya Narayana, and S. Venkataramana, "Effects of the chemical reaction and radiation absorption on an unsteady MHD convective heat and mass transfer flow past a semi-infinite vertical permeable moving plate embedded in a porous medium with heat source and suction," Journal of Applied Mathematics and Mechanics, vol. 7, no. 1, pp. 52-69, 2011.

[24] K. Vajravelu, S. Sreenadh, and P. Lakshminarayana, “The influence of heat transfer on peristaltic transport of a Jeffrey fluid in a vertical porous stratum," Communications in Nonlinear Science and Numerical Simulation, vol. 16, no. 8, pp. 3107-3125, 2011.

[25] A. Y. Bakier and R. S. R. Gorla, "Thermal radiation effect on mixed convection from horizontal surfaces in saturated porous media," Transport in Porous Media, vol. 23, no. 3, pp. 357-363, 1996.

[26] A. Y. Bakier, "Thermal radiation effect on mixed convection from vertical surfaces in saturated porous media," International Communications in Heat and Mass Transfer, vol. 28, no. 1, pp. 119-126, 2001.

[27] M. A. Hossain and H. S. Takhar, "Radiation effect on mixed convection along a vertical plate with uniform surface temperature," Heat and Mass Transfer, vol. 46, pp. 1751-1758, 1996.

[28] C. E. Schwartz and J. M. Smith, "Flow distribution in packed beds," Industrial and Engineering Chemistry, vol. 45, no. 6, pp. 1209-1218, 1969.

[29] W. M. Schertz and K. B. Bischoff, "Thermal and material transport in nonisothermal packed beds," AIChE Journal, vol. 15, no. 4, pp. 597-604, 1969.

[30] R. F. Benenati and C. R. Brosilow, "Void fraction distribution in beds of spheres," AIChE Journal, vol. 8, no. 3, pp. 359-361, 1962. 
[31] B. C. Chandrasekhara and P. M. S. Namboodiri, "Influence of variable permeability on combined free and forced convection about inclined surfaces in porous media," International Journal of Heat and Mass Transfer, vol. 28, no. 1, pp. 199-206, 1985.

[32] S. N. Murthy and J. Feyen, "Influence of variable permeability on the dispersion of a chemically reacting solute in porous media," International Journal of Engineering Science, vol. 27, no. 12, pp. 1661-1671, 1989.

[33] J. A. Shercliff, A Text Book of Magnetohydrodynamics, Pergamon Press, New York, NY, USA, 1965.

[34] F. S. Ibrahim, A. M. Elaiw, and A. A. Bakr, "Effect of the chemical reaction and radiation absorption on the unsteady MHD free convection flow past a semi infinite vertical permeable moving plate with heat source and suction," Communications in Nonlinear Science and Numerical Simulation, vol. 13, no. 6, pp. 1056-1066, 2008.

[35] M. Sudheer Babu and P. V. Satya Narayana, "Effects of the chemical reaction and radiation absorption on free convection flow through porous medium with variable suction in the presence of uniform magnetic field," JP Journal of Heat and Mass Transfer, vol. 3, pp. 219-234, 2009.

[36] R. A. Mohamed and S. M. Abo-Dahab, "Influence of chemical reaction and thermal radiation on the heat and mass transfer in MHD micropolar flow over a vertical moving porous plate in a porous medium with heat generation," International Journal of Thermal Sciences, vol. 48, no. 9, pp. 1800-1813, 2009. 

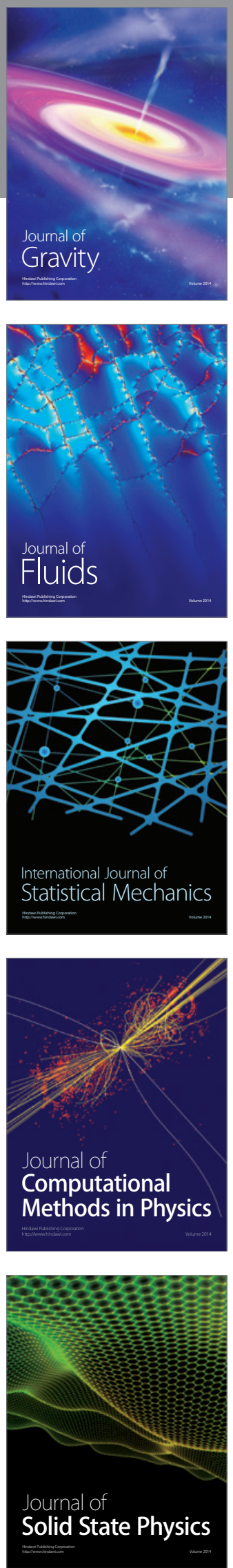

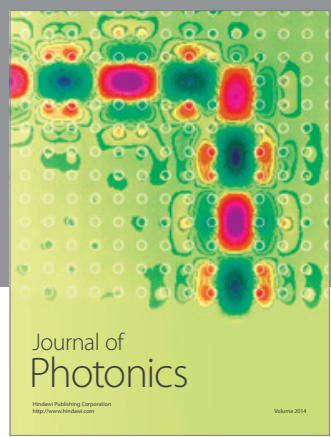

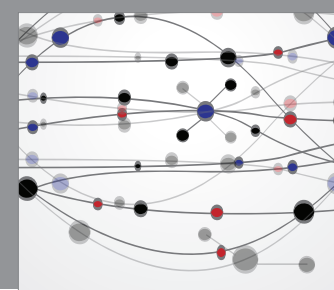

The Scientific World Journal

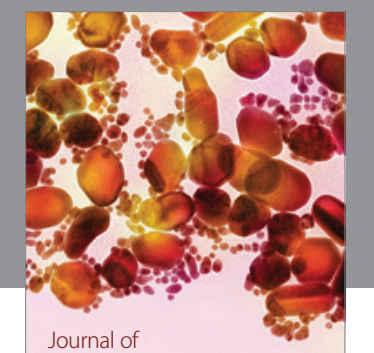

Soft Matter
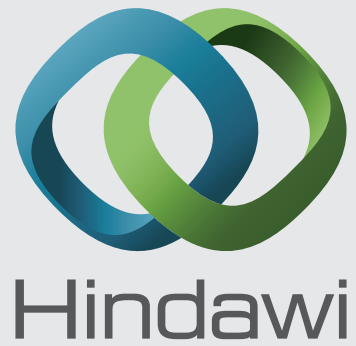

Submit your manuscripts at

http://www.hindawi.com
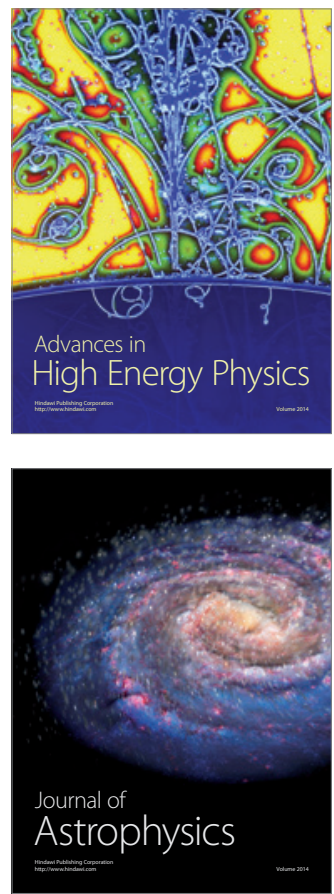
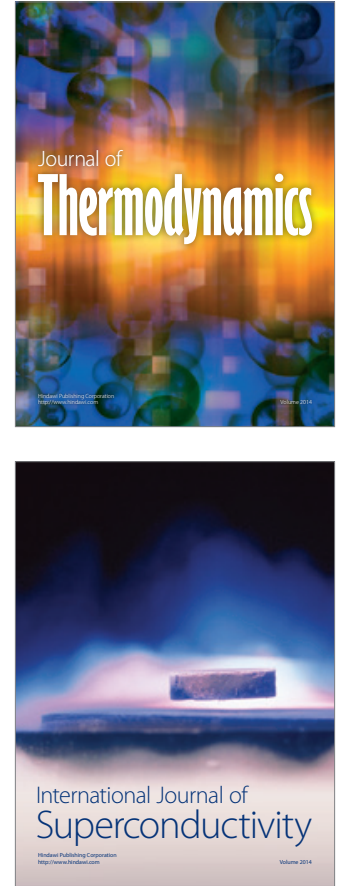
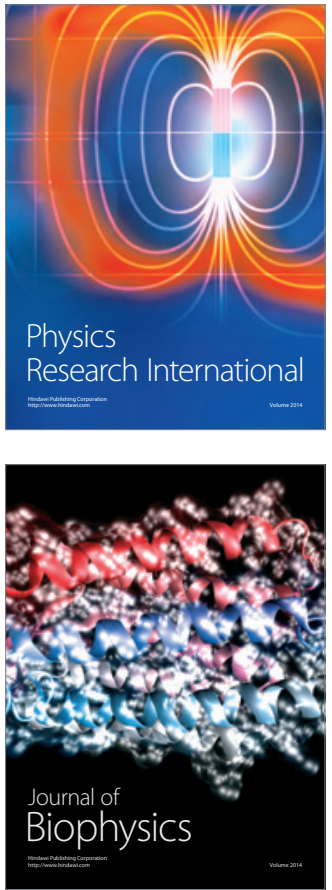
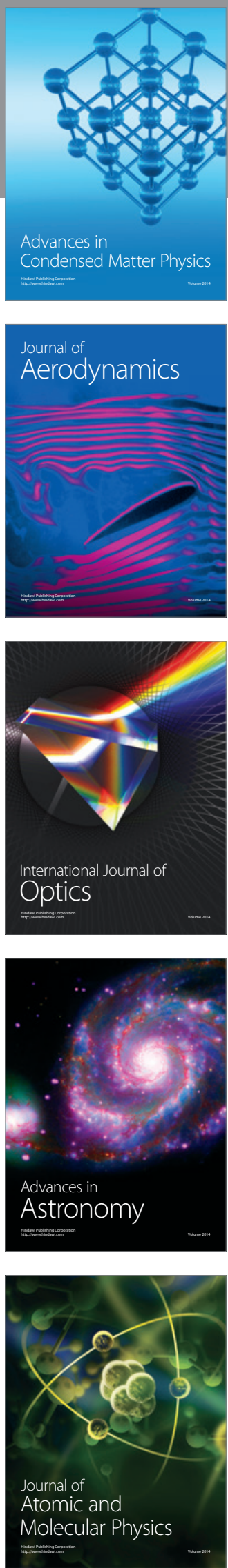